\title{
STOCHASTIC SHELL MODELS DRIVEN BY A MULTIPLICATIVE FRACTIONAL BROWNIAN-MOTION
}

\author{
HAKIMA BESSAIH, MARÍA J. GARRIDO-ATIENZA, AND BJÖRN SCHMALFUSS
}

\begin{abstract}
We prove existence and uniqueness of the solution of a stochastic shell-model. The equation is driven by an infinite dimensional fractional Brownian-motion with Hurst-parameter $H \in(1 / 2,1)$, and contains a non-trivial coefficient in front of the noise which satisfies special regularity conditions. The appearing stochastic integrals are defined in a fractional sense. First, we prove the existence and uniqueness of variational solutions to approximating equations driven by piecewise linear continuous noise, for which we are able to derive important uniform estimates in some functional spaces. Then, thanks to a compactness argument and these estimates, we prove that these variational solutions converge to a limit solution, which turns out to be the unique pathwise mild solution associated to the shell-model with fractional noise as driving process.
\end{abstract}

JANUARY 20, 2016

\section{INTRODUCTION}

In this paper we consider some shell-models under the influence of a noise. Shell-models of turbulence describe the evolution of complex Fourier-like components of a scalar velocity field $u_{n}(t) \in \mathbb{C}$ and the associated wavenumbers $k_{n}$, where the discrete index $n$ is referred as the shell-index. The evolution of the infinite sequence $\left(u_{n}\right)_{n \in \mathbb{N}}$ is given by

$$
\dot{u}_{n}(t)+\nu k_{n}^{2} u_{n}(t)+b_{n}(u(t), u(t))=g_{n}(t, u(t)) \dot{\omega}(t), \quad n \in \mathbb{N}
$$

with the constraints $u_{-1}(t)=u_{0}(t)=0$ and $u_{n}(t) \in \mathbb{C}$ for $n \in \mathbb{N}$. $\dot{\omega}$ denotes a noise path that will be described below. Here $\nu \geq 0$ and, in analogy with Navier-Stokes-equations, $\nu$ represents a kinematic viscosity; $k_{n}=k_{0} \lambda^{n}\left(k_{0}>0\right.$ and $\left.\lambda>1\right)$ and $g_{n}$ is a forcing term. The exact form of $b_{n}(u, v) \in \mathbb{C}$ varies from one model to another. However in all various models, it is assumed that $b_{n}(u, v)$ is chosen in such a way that

$$
\Re \sum_{n=1}^{\infty} b_{n}(u, v) \bar{v}_{n}=0,
$$

where $\Re$ denotes the real part and $\bar{x}$ the complex conjugate of $x$. Equation (1.2) implies a formal law of conservation of energy in the inviscid $(\nu=0)$ and unforced form of (1.1). In particular, we define the bilinear terms $b_{n}$ as

$$
b_{n}(u, v)=i\left(a k_{n+1} \bar{u}_{n+1} \bar{v}_{n+2}+b k_{n} \bar{u}_{n-1} \bar{v}_{n+1}-a k_{n-1} \bar{u}_{n-1} \bar{v}_{n-2}-b k_{n-1} \bar{u}_{n-2} \bar{v}_{n-1}\right)
$$

in the GOY-model (see [18, 24]) and by

$$
b_{n}(u, v)=-i\left(a k_{n+1} \bar{u}_{n+1} v_{n+2}+b k_{n} \bar{u}_{n-1} v_{n+1}+a k_{n-1} u_{n-1} v_{n-2}+b k_{n-1} u_{n-2} v_{n-1}\right)
$$

in the SABRA-model (see [20]). The two parameters $a, b$ are real numbers and $u=\left(u_{n}\right)_{n \in \mathbb{N}}, v=\left(v_{n}\right)_{n \in \mathbb{N}}$. There are several shell-models in literature, the GOY- and SABRA-models defined above have been introduced in $[18,24,20]$. The viscous version of the GOY- and SABRA-models, well posedness, global regularity of solutions and smooth dependence on the initial data can be found in [7].

In recent years, shell-models of turbulence have attracted a lot of interest for their ability to capture some of the statistical properties of the three-dimensional turbulence while presenting a structure much simpler than the Navier-Stokes-equations. The stochastic version of the GOY-model under the influence of an additive

2000 Mathematics Subject Classification. Primary: ; Secondary:

Key words and phrases. Stochastic PDEs, fractional Brownian-motion, pathwise solutions, fractional calculus. 
white noise has been studied in [1] where some statistical properties in terms of the invariant measure have been shown. For the same model in [2,3] a Gaussian invariant measure is associated and a flow constructed.

The introduction of randomness in the Shell model arises from a need to understand the velocity fluctuations observed under identical experimental conditions. Random body forces also arise as control terms, or from random disturbances such as structural vibrations that act on the fluid. In the case of the Navier-Stokes equations, Kolmogorov was the first to introduce white noise in the Navier-Stokes equations in order to obtain an invariant measure for the system. In this article we consider a long term multiplicative noise allowing us to model some memory effects. Such a noise is given by a trace-class fractional Brownian-motion in our state space with Hurst-parameter $H \in(1 / 2,1)$. Our motivation in studying this problem comes from the fact that the Hurst parameter might be used in studying or modeling the exponent of the associated structure functions. A first attempt can be found in [1] on a dyadic model by using a white noise. In addition to noise memory length, fractional Brownian-motion is self-similar with parameter $H$, and when combining this with possible scaling in the space parameter, other regularity properties can be realized, see [12] for details. We hope to perform in the near future some numerical simulations on this model around this question. On the mathematical side, in contrast to white noise, a fractional-Brownian motion is not a martingale, and therefore the multiplicative noise term cannot be presented by an Itô-integral. However, to deal with stochastic integration where the integrator is only Hölder-continuous with an exponent larger than 1/2, one can use the Young-integration, see Young [31] or the adaptation to a stochastic set up by Zähle [32]. Since the definition of these integrals is based on fractional derivatives (see Samko et al. [26] for a general presentation), this theory is often called fractional calculus. An advantage of this theory is, and in contrast to the Itô-integral which is given in general by a limit in probability of Darboux-sums derived from an adapted integrand, that we can define our integral pathwise which means that for any sufficiently regular integrand and integrator the integral is well defined. Or in other words, the exceptional sets of measure zero which appear in the classical Itô-integration does not depend on the integrand. Moreover, integrals can be defined for non-adapted integrands.

The main issue of our work is to prove existence and uniqueness of a pathwise solution of the stochastic shellmodel driven by a fractional multiplicative noise. Applying an infinite dimensional version of the fractional integration theory we are able to present (1.1) in a mild sense where the last term of this equation defines a fractional integral. In particular, the properties of the nonlinear term $B$ generated by the sequence $\left(b_{i}(u, v)\right)_{i \in \mathbb{N}}$ allow to present such a solution in a mild form. Nevertheless, in a first step we replace the fractional noise path by a piecewise linear continuous approximation. Considering (1.1) with such a noise path, we are able to construct global and unique mild solutions. It is important to emphasize that the classical contraction method cannot be used alone since the bilinear term $\left(b_{i}(u, v)\right)_{i \in \mathbb{N}}$ causes to have estimates that do not close with the right norms. This is why we have first to construct weak solutions and get some a priori estimates. These weak solutions have to be constructed with a smoother path noise in order to define the corresponding stochastic integral. The a priori estimates combined with the estimates obtained from the mild form are then used to pass to the limit by means of a compactness argument, and the limit will turn out to be a mild solution of the original problem. The uniqueness of solutions is proved by an argument that uses the balance of suitable norms. As we mentioned before, just using the mild form in its usual norm does not allow to close the estimates. For that reason we combine the a priori estimates and the norms obtained from the mild form to solve an algebraic system of two inequalities where the unknown is given in terms of the difference of two mild solutions starting from the same initial condition but in two different norms. The solution of this system is zero and this is what allows us to conclude the uniqueness of solutions. We believe that our result on existence of solutions can be generalized to the Navier-Stokes equations although careful calculations have to be performed on the nonlinear term which is the main difference with the current result. We might have to work in slightly different spaces, and this will be done in the forthcoming paper [4].

Articles dealing with pathwise solutions for quite general stochastic ordinary differential equations driven by a multiplicative fractional-Brownian motion are, e.g., [22] and [17]. In the infinite dimensional context, there are also articles studying the existence of pathwise solutions, like [23] (dealing with variational solutions) and [21], [16], [10] and [6], for the mild solution. In these papers the Hurst-parameter $H \in(1 / 2,1)$, the diffusion and the drift are assumed to be Lipschitz-continuous and the existence of solutions is proved using pathwise arguments through the fractional integrals. On the other hand, there is an extensive literature for fluid flows 
driven by a Brownian-motion but only a few papers where the noise is a fractional Brownian-motion. For instance, in [5] the considered fractional Brownian-motion has Hurst-parameter bigger than 1/2, and the authors find a local solution of the 3D Navier-Stokes-equation by using the Young-integral. In [12] the 2D Navier-Stokes-equation driven by a fractional Brownian-motion with no restriction on the values of the Hurst-parameter is studied, but the considered noise is additive.

An interesting advantage of considering the existence of pathwise solutions for the stochastic shell-model is that they will generate a random dynamical system, which gives us the possibility to an intensive asymptotic analysis of (1.1). In particular, this is the foundation to show the existence of random attractors and the analysis of their structure. In the forthcoming paper [4] the dynamics of the stochastic shell-model is investigated by using the random dynamical system theory. The generation of a random dynamical system as well as the study of the corresponding random attractor for another kind of stochastic evolution equations with multiplicative fractional noise have been very recently investigated in the papers [6], [15], and [17].

We also would like to point out that, despite the fact that there are similarities between the 2D Navier-Stokesequation and the shell-model, more effort and more involved techniques will be necessary to obtain similar results for the stochastic 2D Navier-Stokes-equation than the ones proved in this paper.

The paper is organized as follows: in Section 2 we introduce the functional analytical framework. In Section 3, we define the fractional derivatives and the stochastic integral using some type of generalized Young-integrals. In Section 4, we introduce the different assumptions on the diffusion and give the definitions of the different solutions. In Section 5, we prove that the system driven by an smoother path has a unique weak solution, that it is also a mild solution. Furthermore, we obtain some fundamental uniform estimates for the solution of the system driven by such a kind of smooth path. In Section 6, thanks to these uniform estimates and a compactness reasoning we construct a unique pathwise mild solution to the shell-model having a fractional Brownian-motion as driving path. Section 7 is devoted to an example of a particular diffusion fitting the assumptions required for developing the abstract framework. Finally, Section 8 contains the proofs of some results that have been used in different sections of the paper.

As usual we denote by $c$ a positive constant that can change their value from line to line.

\section{PRELiminaries}

2.1. Spaces and operators. For any $\alpha \in \mathbb{R}$, let us introduce the following spaces, see Constantin et al. [7] for the details,

$$
V_{\alpha}=\left\{u=\left(u_{1}, u_{2}, \ldots\right) \in \mathbb{C}^{\infty}: \sum_{n=1}^{\infty} k_{n}^{4 \alpha}\left|u_{n}\right|^{2}<\infty\right\}^{1},
$$

where we remind that $\left(k_{n}\right)_{n \in \mathbb{N}}$ are the associated wavenumbers to $\left(u_{n}\right)_{n \in \mathbb{N}}$. The space $V_{\alpha}$ is a separable Hilbert-space with scalar product $(u, v)_{V_{\alpha}}=\sum_{n=1}^{\infty} k_{n}^{4 \alpha} u_{n} \bar{v}_{n}$. Denote by $\|\cdot\|_{V_{\alpha}}$ its norm. We have the compact embedding

$$
V_{\alpha_{1}} \subset V_{\alpha_{2}} \quad \text { if } \alpha_{1}>\alpha_{2} \text {. }
$$

Let us denote by $V:=V_{0}$ and its norm simply by $\|\cdot\|$ and its scalar product by $(\cdot, \cdot)_{V}$.

Let $A: D(A)=V_{1} \rightarrow V$ be the linear unbounded operator defined as

$$
A:\left(u_{1}, u_{2}, \ldots\right) \mapsto\left(-\nu k_{1}^{2} u_{1},-\nu k_{2}^{2} u_{2}, \ldots\right) .
$$

For simplicity let us set $\nu=1$. It is known that $A$ generates an analytic semigroup $S(\cdot)$ which follows from the Lax-Milgram lemma, see Sell and You [27] Theorem 36.6, and this semigroup is exponentially stable. Furthermore, $V_{\alpha}=D\left(A^{\alpha}\right)$ and $(u, v)_{V_{\alpha}}=\left(A^{\alpha} u, A^{\alpha} v\right)_{V}, u, v \in V_{\alpha}$.

Let $L\left(V_{\delta}, V_{\gamma}\right)$ denote the space of linear continuous operators from $V_{\delta}$ into $V_{\gamma}$. As usual, $L(V)$ denotes $L(V, V)$. The following properties are well known for analytic semigroups and their generators: for $\zeta \geq \alpha$ there exists a constant $c>0$ such that

$$
\begin{gathered}
|S(t)|_{L\left(V_{\alpha}, V_{\zeta}\right)}=\left|A^{\zeta} S(t)\right|_{L\left(V_{\alpha}, V\right)} \leq \frac{c}{t^{\zeta-\alpha}} e^{-\lambda t}, \quad t>0, \\
|S(t)-\mathrm{id}|_{L\left(V_{\sigma+\nu}, V_{\theta+\nu}\right)} \leq c t^{\sigma-\theta}, \quad \text { for } \sigma \in[\theta, 1+\theta], \quad \nu \in \mathbb{R},
\end{gathered}
$$

\footnotetext{
${ }^{1}$ Here there is an important difference w.r.t. the notation of spaces in [7] and [8].
} 
where $\lambda$ in (2.1) is a positive constant, see for instance Pazy [25] Theorem 2.6.13. From these inequalities, for $\nu, \eta \in[0,1], \zeta, \delta \in \mathbb{R}$ such that $\delta \leq \zeta+\nu$, there exists a $c>0$ such that for $0 \leq q \leq r \leq s \leq t$,

$$
\begin{array}{r}
|S(t-r)-S(t-q)|_{L\left(V_{\delta}, V_{\zeta}\right)} \leq c(r-q)^{\nu}(t-r)^{-\nu-\zeta+\delta}, \\
|S(t-r)-S(s-r)-S(t-q)+S(s-q)|_{L(V)} \leq c(t-s)^{\nu}(r-q)^{\eta}(s-r)^{-(\nu+\eta)} .
\end{array}
$$

Define the bilinear operator $B: \mathbb{C}^{\infty} \times \mathbb{C}^{\infty} \rightarrow \mathbb{C}^{\infty}$ as

$$
B(u, v)=-\left(b_{1}(u, v), b_{2}(u, v), \ldots\right)
$$

where the components $b_{n}$ satisfy (1.2).

$B$ is well defined when its domain is $V_{1 / 2} \times V$ or $V \times V_{1 / 2}$ (see [7]), that is, $B: V_{1 / 2} \times V \rightarrow V$ and $B$ : $V \times V_{1 / 2} \rightarrow V$ are bounded operators. The operator $B$ enjoys the following properties

$$
\begin{array}{ll}
\Re(B(u, v), w)_{V}=-\Re(B(u, w), v)_{V}, & u \in V_{1 / 2}, \quad v, w \in V, \\
\Re(B(u, v), w)_{V}=-\Re(B(u, w), v)_{V}, & u \in V, \quad v, w \in V_{1 / 2} .
\end{array}
$$

As a consequence, we also have that

$$
\Re(B(u, v), v)_{V}=0, \quad u \in V, \quad v \in V_{1 / 2} .
$$

Moreover, we extend the result of Constantin et al. [7] to more general spaces:

Lemma 2.1. For any $\alpha_{1}, \alpha_{2}, \alpha_{3} \in \mathbb{R}$

$$
B: V_{\alpha_{1}} \times V_{\alpha_{2}} \rightarrow V_{-\alpha_{3}} \text { with } \alpha_{1}+\alpha_{2}+\alpha_{3} \geq \frac{1}{2}
$$

and there exists a constant $c$ depending on the $\alpha_{j}$ 's such that

$$
\|B(u, v)\|_{V_{-\alpha_{3}}} \leq c\|u\|_{V_{\alpha_{1}}}\|v\|_{V_{\alpha_{2}}}, \quad u \in V_{\alpha_{1}}, \quad v \in V_{\alpha_{2}} .
$$

The proof of this result follows by Proposition 1 of Constantin et al. [8], and Bessaih and Ferrario [2] and hence we omit it here.

Let $C\left([s, t] ; V_{\mu}\right)$ be the space of continuous functions on $[s, t]$ with values in $V_{\mu}$ and with the usual norm $\|\cdot\|_{C, \mu}$ (or $\|\cdot\|_{C, s, t, \mu}$ when we want to stress the interval). In the particular case that $\mu=0$, we simply write $\|\cdot\|_{C}$ (or $\|\cdot\|_{C, s, t}$ respectively). For $\beta \in(0,1]$ we denote by $C^{\beta}\left([s, t] ; V_{\mu}\right)$ the space of Hölder-continuous functions on $[s, t]$ and with values in $V_{\mu}$, equipped with the norm

$$
\|u\|_{\beta, \mu}=\|u\|_{C, \mu}+\|\| u\|\|_{\beta, \mu}, \quad\|\| u \|_{\beta, \mu}:=\sup _{s \leq p<q \leq t} \frac{\|u(q)-u(p)\|_{V_{\mu}}}{(q-p)^{\beta}} .
$$

In particular, for the case $\beta=1$ this is the space of Lipschitz-continuous functions.

The spaces $L^{p}\left(s, t ; V_{\mu}\right), p \in[1, \infty]$ have the standard meaning with the usual norms.

As we have mentioned above, sometimes it is important to consider the above norms on different time intervals $[s, t]$, thus in those cases the time interval will be indicated in the index of the norm.

For the previous spaces the following compactness theorem holds true:

Theorem 2.2. (i) For $\alpha>0$ and $\delta \in \mathbb{R}, L^{2}\left(s, t ; V_{\alpha}\right) \cap C^{\beta}\left([s, t] ; V_{-\delta}\right)$ is compactly embedded into $L^{2}(s, t ; V) \cap$ $C\left([s, t] ; V_{-\delta}\right)$.

(ii) For $0 \leq \delta_{1}<\delta_{2}$ and $0 \leq \beta_{1}<\beta_{2} \leq 1$ the space $C^{\beta_{2}}\left([s, t] ; V_{-\delta_{1}}\right)$ is compactly embedded into $C^{\beta_{1}}\left([s, t] ; V_{-\delta_{2}}\right)$.

For the first part see Vishik and Fursikov [30] Chapter IV Theorem 4.1. For the second part we refer to Maslowski and Nualart [21] Lemma 4.5. Indeed, we have the compact embedding $V_{-\delta_{1}} \subset V_{-\delta_{2}}$.

For $t \in[0, T]$, we now rewrite the equation (1.1) in an abstract form,

$$
d u(t)=(A u(t)+B(u(t), u(t))) d t+G(u(t)) d \omega(t), \quad u(0)=u_{0},
$$

where $G$ is a nontrivial diffusion term representing the external force, that satisfies certain assumptions which are given in Section 4 below. Here $\omega$ represents a path in $C^{\beta^{\prime}}([0, T] ; V)$, with $\beta^{\prime}>1 / 2$, or in particular, a fractional Brownian-motion with Hurst-parameter $H \in(1 / 2,1)$, see the definition in Section 3. The corresponding stochastic evolution equation has therefore a multiplicative noise. In what follows we will 
describe the type of stochastic integral we are going to consider, which will allow us to give an appropriate meaning to $(2.5)$.

\section{Integrals in HilberT-SPACES FOR HÖLDER-CONTINUOUS INTEGRATORS with HÖLDER EXPONENTS GREATER THAN $1 / 2$}

In this section we are concerned with the definition of the following infinite dimensional integral

$$
\int_{T_{1}}^{T_{2}} Z d \omega
$$

where $\omega$ is a Hölder-continuous function with Hölder exponent $\beta^{\prime}>1 / 2$ and $Z$ is an appropriate integrand. We follow the recent definition given by Chen et al. [6], and for the sake of completeness, next we shall borrow the main steps of their construction.

We start by considering an abstract separable Hilbert-space $\tilde{V}$, then for $0<\alpha<1$ and general measurable functions $Z:\left[T_{1}, T_{2}\right] \rightarrow \tilde{V}$ and $\omega:\left[T_{1}, T_{2}\right] \rightarrow V$, we define the following fractional derivatives

$$
\begin{aligned}
D_{T_{1}+}^{\alpha} Z[r] & =\frac{1}{\Gamma(1-\alpha)}\left(\frac{Z(r)}{\left(r-T_{1}\right)^{\alpha}}+\alpha \int_{T_{1}}^{r} \frac{Z(r)-Z(q)}{(r-q)^{1+\alpha}} d q\right) \in \tilde{V}, \\
D_{T_{2}-}^{1-\alpha} \omega_{T_{2}-}[r] & =\frac{(-1)^{1-\alpha}}{\Gamma(\alpha)}\left(\frac{\omega(r)-\omega\left(T_{2}-\right)}{\left(T_{2}-r\right)^{1-\alpha}}+(1-\alpha) \int_{r}^{T_{2}} \frac{\omega(r)-\omega(q)}{(q-r)^{2-\alpha}} d q\right) \in V,
\end{aligned}
$$

where $\omega_{T_{2}-}(r)=\omega(r)-\omega\left(T_{2}-\right)$, being $\omega\left(T_{2}-\right)$ the left-sided limit of $\omega$ at $T_{2}$. Here $\Gamma(\cdot)$ denotes the Gamma function.

Let us start with the case when the integrand $z$ and the integrator $\zeta$ are one-dimensional. Suppose that $z\left(T_{1}+\right), \zeta\left(T_{1}+\right), \zeta\left(T_{2}-\right)$ exist, being respectively the right-sided limit of $z$ at $T_{1}$ and the right- and left-sided limits of $\zeta$ at $T_{1}, T_{2}$, and that $z \in I_{T_{1}+}^{\alpha}\left(L^{p}\left(T_{1}, T_{2} ; \mathbb{R}\right)\right), \zeta_{T_{2}-} \in I_{T_{2}-}^{1-\alpha}\left(L^{p^{\prime}}\left(T_{1}, T_{2} ; \mathbb{R}\right)\right)$ with $1 / p+1 / p^{\prime} \leq 1$ and $\alpha p<1$ (the definition of these spaces can be found, for instance, in Samko et al. [26]). Then following Zähle [32] we define

$$
\int_{T_{1}}^{T_{2}} z d \zeta=(-1)^{\alpha} \int_{T_{1}}^{T_{2}} D_{T_{1}+}^{\alpha} z[r] D_{T_{2}-}^{1-\alpha} \zeta_{T_{2}-}[r] d r .
$$

Suppose now that $\zeta$ is Lipschitz-continuous. Then $\zeta$ generates a signed measure $d \zeta$ and $\zeta \in I_{T_{2}-}^{\alpha}\left(L^{p^{\prime}}\left(T_{1}, T_{2} ; \mathbb{R}\right)\right)$. Therefore, in this situation the integral

$$
\int_{T_{1}}^{T_{2}} z d \zeta
$$

can be expressed by (3.1).

Let $\hat{V}$ be a separable Hilbert-space endowed with the norm $\|\cdot\|_{\hat{V}}$ and consider the separable Hilbert-space $L_{2}(V, \hat{V})$ of Hilbert-Schmidt-operators from $V$ into $\hat{V}$ with the norm $\|\cdot\|_{L_{2}(V, \hat{V})}$ and inner product $(\cdot, \cdot)_{L_{2}(V, \hat{V})}$. Let $\left(e_{i}\right)_{i \in \mathbb{N}}$ and $\left(f_{i}\right)_{i \in \mathbb{N}}$ be a complete orthonormal basis of $V$ and $\hat{V}$, resp. A base in $L_{2}(V, \hat{V})$ is given by

$$
E_{i j} e_{k}=\left\{\begin{array}{lll}
0 & : & j \neq k \\
f_{i} & : & j=k
\end{array}\right.
$$

Let us consider now mappings $Z:\left[T_{1}, T_{2}\right] \rightarrow L_{2}(V, \hat{V})$ and $\omega:\left[T_{1}, T_{2}\right] \rightarrow V$. Suppose that $z_{j i}=\left(Z, E_{j i}\right)_{L_{2}(V, \hat{V})} \in$ $I_{T_{1}+}^{\alpha}\left(L^{p}\left(T_{1}, T_{2} ; \mathbb{R}\right)\right)$ and $z_{j i}\left(T_{1}+\right)$ exists and $\alpha p<1$. Moreover, let us also assume that $\zeta_{i T_{2}-}=\left(\omega_{T_{2}-}(t), e_{i}\right)_{V} \in$ $I_{T_{2}-}^{1-\alpha}\left(L^{p^{\prime}}\left(T_{1}, T_{2} ; \mathbb{R}\right)\right)$ such that $1 / p+1 / p^{\prime} \leq 1$, and the mapping

$$
\left[T_{1}, T_{2}\right] \ni r \mapsto\left\|D_{T_{1}+}^{\alpha} Z[r]\right\|_{L_{2}(V, \hat{V})}\left\|D_{T_{2}-}^{1-\alpha} \omega_{T_{2}-}[r]\right\| \in L^{1}\left(T_{1}, T_{2} ; \mathbb{R}\right) .
$$

We introduce

$$
\begin{aligned}
\int_{T_{1}}^{T_{2}} Z d \omega & :=(-1)^{\alpha} \int_{T_{1}}^{T_{2}} D_{T_{1}+}^{\alpha} Z[r] D_{T_{2}-}^{1-\alpha} \omega_{T_{2}-}[r] d r \\
& :=(-1)^{\alpha} \sum_{j=1}^{\infty}\left(\sum_{i=1}^{\infty} \int_{T_{1}}^{T_{2}} D_{T_{1}+}^{\alpha} z_{j i}[r] D_{T_{2}-}^{1-\alpha} \zeta_{i T_{2}-}[r] d r\right) f_{j}
\end{aligned}
$$


This last equality is well defined due to the fact that Pettis' theorem and the separability of $V$ ensure that the integrand is weakly measurable and hence measurable. Moreover, the norm of the above integral is given by

$$
\begin{aligned}
\left\|\int_{T_{1}}^{T_{2}} Z d \omega\right\|_{\hat{V}} & =\left(\sum_{j=1}^{\infty}\left|\sum_{i=1}^{\infty} \int_{T_{1}}^{T_{2}} D_{T_{1}+}^{\alpha} z_{j i}[r] D_{T_{2}-}^{1-\alpha} \zeta_{i T_{2}-}[r] d r\right|^{2}\right)^{\frac{1}{2}} \\
& \leq \int_{T_{1}}^{T_{2}}\left\|D_{T_{1}+}^{\alpha} Z[r]\right\|_{L_{2}(V, \hat{V})}\left\|D_{T_{2}-}^{1-\alpha} \omega_{T_{2}-}[r]\right\| d r .
\end{aligned}
$$

The next result, which proof can be found in [6], considers the definition of the above integral when having suitable Hölder continuous integrator and integrand functions:

Lemma 3.1. Suppose that $0 \leq T_{1}<T_{2}, Z \in C^{\beta}\left(\left[T_{1}, T_{2}\right] ; L_{2}(V, \hat{V})\right)$ and $\omega \in C^{\beta^{\prime}}\left(\left[T_{1}, T_{2}\right] ; V\right)$ with $1-\beta^{\prime}<$ $\alpha<\beta$. Then

$$
\int_{T_{1}}^{T_{2}} Z d \omega \in \hat{V}
$$

is well-defined in the sense of (3.2). Also, there exists a constant $c$ depending on $T_{2}-T_{1}, \alpha, \beta$ and $\beta^{\prime}$ such that

$$
\left\|\int_{T_{1}}^{T_{2}} Z d \omega\right\|_{\hat{V}} \leq c\|Z\|_{C^{\beta}\left(\left[T_{1}, T_{2}\right] ; L_{2}(V, \hat{V})\right)}\|\omega \mid\| \|_{\beta^{\prime}, T_{1}, T_{2}}\left(T_{2}-T_{1}\right)^{\beta^{\prime}} .
$$

Moreover, the above integral with driving path $\omega$ is well-defined even though the integrand is locally Höldercontinuous, which will be the case in the next sections when the semigroup $S$ is part of the integrand, see [6] for the proof of this assertion.

In the following we would like to consider the above integrals when the integrator is a noise given by a fractional Brownian-motion ( $\mathrm{fBm})$. An one-dimensional $\mathrm{fBm}$ is a centered Gaussian-process given by the auto-covariance

$$
R(s, t)=\frac{1}{2}\left(t^{2 H}+s^{2 H}-|t-s|^{2 H}\right)
$$

where $H \in(0,1)$ is the so-called Hurst-parameter. The value $H=1 / 2$ determines a Brownian-motion, which is a martingale and a Markov-process with independent increments. When $H \neq 1 / 2$ these properties do not hold.

An $\mathrm{fBm}$ can be also defined in a separable Hilbert-space. By the following construction we obtain such an infinite-dimensional noise with values in $V$ : let $\left(\zeta_{i}\right)_{i \in \mathbb{N}}$ be a iid-sequence of $\mathrm{fBm}$ in $\mathbb{R}$ having the same Hurst-parameter $H$. Then

$$
t \rightarrow \omega(t):=\sum_{i=1}^{\infty} q_{i}^{\frac{1}{2}} \zeta_{i} e_{i}
$$

where $\left(q_{i}\right)_{i \in \mathbb{N}} \in l_{2}$, defines an fBm with values in $V$ and with auto-covariance

$$
\frac{1}{2} Q\left(t^{2 H}+s^{2 H}-|t-s|^{2 H}\right)
$$

where the operator $Q$ of diagonal form is defined by

$$
\left(e_{i}, Q e_{j}\right)_{V}=\delta_{i j} q_{i} .
$$

For simplicity we restrict ourselves to a real fBm. However, taking two one-dimensional independent real fBm $\zeta^{1}, \zeta^{2}$ then we could construct a one-dimensional complex fBm: $\zeta:=1 / \sqrt{2}\left(\zeta^{1}+i \zeta^{2}\right)$. Then by the above formula we could construct a complex fBm $\omega$ in $V$.

Remark 3.2. For our further purposes we need the $f B m \omega$ to be piecewise linearly approximated. As one can check later, we will use the property that given $\omega$ we can find a sequence of piecewise linear continuous functions $\omega_{n}$ converging to $\omega$ in a Hölder-continuous space. However, the space of Hölder-continuous functions is not separable, but we can modify it in such a way that the modified space is: for $\gamma \in(0,1)$, the space

$$
C^{0, \gamma}([0, T] ; V):=\left\{\omega \in C^{\gamma}([0, T] ; V): \lim _{\delta \rightarrow 0} \sup _{\left|s_{1}-s_{2}\right|<\delta,[0, T] \ni s_{1} \neq s_{2}} \frac{\left\|\omega\left(s_{1}\right)-\omega\left(s_{2}\right)\right\|}{\left(s_{1}-s_{2}\right)^{\gamma}}=0\right\},
$$


is a separable space since $V$ is itself separable (see [13], [14] and [11]). On the other hand, thanks to Kolmogorov's theorem (see Theorem 1.4.1 in Kunita [19]), it is known that if $\omega$ represents an fBm with Hurst parameter $H$, then there exists a version, that we also call $\omega$, such that $\omega \in C^{\beta^{\prime}}([0, T] ; V)$ for any $\beta^{\prime}<H$. Let us take $\gamma<\beta^{\prime}<H$, then it is easy to see that $C^{\beta^{\prime}}([0, T] ; V) \subset C^{0, \gamma}([0, T] ; V)$, and therefore this latter space is the one that we should take when the aim is to approximate the fBm. However, for the easy of presentation, in what follows we will assume that $\omega$ can be piecewise linearly approximated by a sequence $\left(\omega_{n}\right)_{n \in \mathbb{N}}$ converging in $C^{\beta^{\prime}}([0, T] ; V)$, but this statement must be understood according to the sense given above.

\section{Definition of a SOlution of the Stochastic SHell-MOdel}

In this section we would like to formulate conditions ensuring that (2.5) has a unique global solution.

We emphasize that we have to give a definition of solution which is appropriate in our context: on the one hand, the driving function belongs to $C^{\beta^{\prime}}([0, T] ; V)$ for a $\beta^{\prime} \in(1 / 2,1)$, which means that we cannot define integrals by using the standard integration theory of bounded-variation integrators. In particular our situation here covers the case when the driving function is given by an $\mathrm{fBm}$ in $V$ with Hurst-parameter $H>1 / 2$. On the other hand, the bilinear operator $B$ is unbounded and not Lipschitz-continuous in this model.

We now give the assumptions for the diffusion operator $G$ of (2.5). In what follows, we choose a constant $\delta>0$ which will be determined later, and by $L_{2}(V)$ we denote the space $L_{2}(V, V)$.

Assumption (G) Assume that the mapping $G: V_{-\delta} \rightarrow L_{2}(V)$ is bounded and twice continuously Fréchetdifferentiable with bounded first and second derivatives $D G(u)$ and $D^{2} G(u)$, for $u \in V_{-\delta}$. Let us denote, respectively, by $c_{G}, c_{D G}$ and $c_{D^{2} G}$ the bounds for $G, D G$ and $D^{2} G$. Then, for $u \in V_{-\delta}$

$$
\|G(u)\|_{L_{2}(V)} \leq c_{G} .
$$

Furthermore, for $u_{1}, u_{2} \in V_{-\delta}$,

$$
\left\|G\left(u_{1}\right)-G\left(u_{2}\right)\right\|_{L_{2}(V)} \leq c_{D G}\left\|u_{1}-u_{2}\right\|_{V_{-\delta}},
$$

and for $u_{1}, u_{2}, v_{1}, v_{2} \in V_{-\delta}$,

$$
\begin{aligned}
& \left\|G\left(u_{1}\right)-G\left(v_{1}\right)-\left(G\left(u_{2}\right)-G\left(v_{2}\right)\right)\right\|_{L_{2}(V)} \\
& \quad \leq c_{D G}\left\|u_{1}-v_{1}-\left(u_{2}-v_{2}\right)\right\|_{V_{-\delta}}+c_{D^{2} G}\left\|u_{1}-u_{2}\right\|_{V_{-\delta}}\left(\left\|u_{1}-v_{1}\right\|_{V_{-\delta}}+\left\|u_{2}-v_{2}\right\|_{V_{-\delta}}\right) .
\end{aligned}
$$

Notice that $D G: V_{-\delta} \mapsto L_{2}\left(V_{-\delta}, L_{2}(V)\right)=L_{2}\left(V_{-\delta} \times V, V\right)$ is a bilinear mapping whereas $D^{2} G$ a trilinear mapping.

In this paper we shall look at the existence and uniqueness of a solution of (2.5) according to the next definition:

Definition 4.1. Let $1 / 2<\beta<\beta^{\prime} \leq 1$ and let $\omega \in C^{\beta^{\prime}}([0, T] ; V), u_{0} \in V$ and $\delta \in(\beta, 1)$. A function $u$ is said to be a mild solution to (2.5) over the interval $[0, T]$ associated to the initial condition $u_{0}$ if

$$
u \in C([0, T] ; V) \cap L^{2}\left(0, T ; V_{1 / 2}\right) \cap C^{\beta}\left([0, T] ; V_{-\delta}\right)
$$

and such that

$$
u(t)=S(t) u_{0}+\int_{0}^{t} S(t-r) B(u(r), u(r)) d r+\int_{0}^{t} S(t-r) G(u(r)) d \omega(r)
$$

for every $t \in[0, T]$.

Remark 4.2. Note that the first integral in (4.2) is well defined in $V$ because of the fact that $u \in C([0, T] ; V) \cap$ $L^{2}\left(0, T ; V_{1 / 2}\right)$ and Lemma 2.1. The stochastic integral in (4.2) must be understood in $V$ according to the definition given in Section 3.

We stress that we are interested in finding a mild solution for (2.5). Following [23] we could also consider weak solutions for our problem. Nevertheless for $u \in L^{\infty}(0, T ; V) \cap L^{2}\left(0, T ; V_{1 / 2}\right)$ we have that $B(u, u)$ is sufficiently regular so that we can work with mild solutions.

When $\omega$ is regular, we can also interpret the solution in the following weak sense: 
Definition 4.3. Assume that $\omega$ is piecewise linear continuous in $[0, T]$ with values in $V$. We say that $u$ is a weak solution to (2.5) over the interval $[0, T]$ associated to the initial condition $u_{0} \in V$ if

$$
u \in C([0, T] ; V) \cap L^{2}\left(0, T ; V_{1 / 2}\right)
$$

and such that

$$
(u(t), \varphi)_{V}+\int_{0}^{t}\left(A^{1 / 2} u(s), A^{1 / 2} \varphi\right)_{V} d s-\int_{0}^{t}(B(u(s), u(s)), \varphi)_{V} d s=\left(u_{0}, \varphi\right)_{V}+\int_{0}^{t}\left(G(u(s)) \omega^{\prime}(s), \varphi\right)_{V} d s
$$

holds for every $\varphi \in V_{1 / 2}$ and $t \in[0, T]$.

\section{Solutions of the Stochastic Shell-Model For Piecewise Linear Continuous Path Noise}

In this section we assume that $\omega$ is a piecewise linear continuous function. This case is the foundation for studying the more general case which will be treated in the next section. Indeed, in further sections given $\omega \in C^{\beta^{\prime}}([0, T] ; V)$ we shall consider a sequence $\left(\omega_{n}\right)_{n \in \mathbb{N}}$ of piecewise linear continuous paths converging to $\omega \in C^{\beta^{\prime}}([0, T] ; V)$, see Remark 3.2. As we cannot assume that the sequence $\left(\omega_{n}^{\prime}\right)_{n \in \mathbb{N}}$ in uniformly bounded in $L^{\infty}(0, T ; V)$, we will have to construct uniform a priori estimates for the solutions to equations driven by $\omega_{n}$, which will be based on uniform estimates of $\left(\omega_{n}\right)_{n \in \mathbb{N}}$ with respect to the $C^{\beta^{\prime}}$-norm.

We start by studying the existence of solutions for the stochastic Shell-model having this kind of regular driving function:

Proposition 5.1. Let $u_{0} \in V, \omega$ be a piecewise linear continuous function and assume that $G$ satisfies the assumption $(\mathbf{G})$. Then, there is a unique global weak solution $u$ for equation (2.5) in the sense of Definition 4.3.

Proof. The proof is very classical but, for the the sake of completeness, we will sketch it here. Let us denote by $P_{n}$ the projection operator in $V$ onto the space spanned by $e_{1}, e_{2}, \ldots, e_{n}$. Then, the Galerkin-approximations $\left(u_{n}\right)_{n \in \mathbb{N}}$ to problem (2.5) are solutions of the finite-dimensional systems

$$
d u_{n}(t)=\left(A u_{n}(t)+P_{n} B\left(u_{n}(t), u_{n}(t)\right)\right) d t+P_{n} G\left(u_{n}(t)\right) \omega^{\prime}(t) d t .
$$

On the other hand, if $G^{*}$ denotes the adjoint operator of $G$, taking the scalar product of (5.1) by $u_{n}$, using the property (2.4) and the assumption $(\mathbf{G})$, we get that

$$
\begin{aligned}
\frac{1}{2} \frac{d}{d t}\left\|u_{n}(t)\right\|^{2}+\left\|u_{n}(t)\right\|_{V_{1 / 2}}^{2} & \leq\left|\left(P_{n} G\left(u_{n}(t)\right) \omega^{\prime}(t), u_{n}(t)\right)_{V}\right| \leq\left|\left(\omega^{\prime}(t), G^{*}\left(u_{n}(t)\right) u_{n}(t)\right)_{V}\right| \\
& \leq\left\|\omega^{\prime}(t)\right\|\left\|G^{*}\left(u_{n}(t)\right) u_{n}(t)\right\| \leq c_{G}\left\|\omega^{\prime}(t)\right\|\left\|u_{n}(t)\right\| \\
& \leq \frac{c_{G}^{2}}{2}\left\|\omega^{\prime}(t)\right\|^{2}+\frac{1}{2}\left\|u_{n}(t)\right\|^{2} .
\end{aligned}
$$

Hence, using Gronwall's lemma yields that

$$
\sup _{t \in[0, T]}\left\|u_{n}(t)\right\|^{2} \leq c\left(\left\|u_{n}(0)\right\|,\left\|\omega^{\prime}\right\|_{L^{\infty}(0, T ; V)}^{2}, T\right)
$$

for an appropriate positive constant $c$, and consequently we also have

$$
\int_{0}^{T}\left\|u_{n}(t)\right\|_{V_{1 / 2}}^{2} d t \leq c
$$

uniformly in $n$.

Also, by classical arguments, we get that $\left(u_{n}\right)_{n \in \mathbb{N}}$ is bounded in $C^{\hat{\beta}}\left([0, T] ; V_{-\delta}\right)$ for $\delta>1 / 2$ and $\hat{\beta}<1$. In fact, since $u_{n} \in L^{\infty}(0, T ; V)$, it follows by Lemma 2.1

$$
\sup _{0 \leq s<t \leq T} \frac{\int_{s}^{t}\left\|A^{-\delta} B\left(u_{n}(r), u_{n}(r)\right)\right\| d r}{(t-s)^{\hat{\beta}}} \leq c \sup _{0 \leq s<t \leq T} \frac{\int_{s}^{t}\left\|A^{-\frac{1}{2}} B\left(u_{n}(r), u_{n}(r)\right)\right\| d r}{(t-s)^{\hat{\beta}}} \leq c T^{1-\hat{\beta}}\left\|u_{n}\right\|_{L^{\infty}(0, T ; V)}^{2}<\infty,
$$


and by $(\mathbf{G})$ we arrive at

$$
\sup _{0 \leq s<t \leq T} \frac{\int_{s}^{t}\left\|A^{-\delta} G\left(u_{n}(r)\right) \omega^{\prime}(r)\right\| d r}{(t-s)^{\hat{\beta}}} \leq c \sup _{0 \leq s<t \leq T} \frac{\int_{s}^{t}\left\|G\left(u_{n}(r)\right)\right\|_{L_{2}(V)}\left\|\omega^{\prime}(r)\right\| d r}{(t-s)^{\hat{\beta}}} \leq c c_{G} T^{1-\hat{\beta}}\left\|\omega^{\prime}\right\|_{L^{\infty}(0, T ; V)}<\infty .
$$

Moreover, applying the interpolation inequality (see [27], Theorem 37.6), we know that there exists a constant $c=c(\delta) \geq 1$ such that

$$
\left\|A^{1-\delta} v\right\| \leq c\left\|A^{0} v\right\|^{2 \delta-1}\left\|A^{1 / 2} v\right\|^{2-2 \delta} \quad \text { for all } v \in V,
$$

and therefore, for $\hat{\beta} \in(1 / 2,1)$ and $\delta \in(\hat{\beta}, 1)$,

$$
\begin{aligned}
& \sup _{0 \leq s<t \leq T} \frac{\int_{s}^{t}\left\|A^{-\delta} A u_{n}(r)\right\| d r}{(t-s)^{\hat{\beta}}} \leq c \sup _{0 \leq s<t \leq T} \frac{\int_{s}^{t}\left\|u_{n}(r)\right\|^{2 \delta-1}\left\|A^{1 / 2} u_{n}(r)\right\|^{2-2 \delta} d r}{(t-s)^{\hat{\beta}}} \\
& \leq c\left\|u_{n}\right\|_{L^{\infty}(0, T ; V)}^{2 \delta-1} \sup _{0 \leq s<t \leq T} \frac{\left(\int_{s}^{t} d r\right)^{\delta}\left(\int_{s}^{t}\left\|A^{1 / 2} u_{n}(r)\right\|^{2} d r\right)^{1-\delta}}{(t-s)^{\hat{\beta}}} \leq c T^{\delta-\hat{\beta}}\left\|u_{n}\right\|_{L^{\infty}(0, T ; V)}^{2 \delta-1}\left\|u_{n}\right\|_{L^{2}\left(0, T ; V_{1 / 2}\right)}^{2-2 \delta}<\infty .
\end{aligned}
$$

Hence, by the compactness Theorem 2.2 (i) we get a subsequence, still denoted by $\left(u_{n}\right)_{n \in \mathbb{N}}$, that converges strongly in $L^{2}(0, T ; V) \cap C\left([0, T] ; V_{-\delta}\right)$ to some limit $u$. Since $\left(u_{n}\right)_{n \in \mathbb{N}}$ is bounded in $L^{\infty}(0, T ; V) \cap L^{2}\left(0, T ; V_{1 / 2}\right)$ this sequence is relatively weak-star compact in $L^{\infty}(0, T ; V)$ and relatively weak compact in $L^{2}\left(0, T ; V_{1 / 2}\right)$. As a consequence, the limit $u \in L^{\infty}(0, T ; V) \cap L^{2}\left(0, T ; V_{1 / 2}\right)$. Now, it remains to prove that the limit $u$ is a solution to the system (2.5) according to the Definition 4.3. Indeed, assuming that $u_{n}$ is solution in the sense of Definition 4.3, we can pass to the limit on each term. Furthermore, the regularity of $u$ implies that the right hand side of (4.3) as well as the last two terms of the left hand side of $(4.3)$ are in $C([0, T] ; V)$, hence $u \in C([0, T] ; V)$.

Moreover, we have the following result about mild solutions:

Proposition 5.2. Assume the same hypotheses than in Proposition 5.1 and that $\hat{\beta} \in(1 / 2,1), \delta \in(\hat{\beta}, 1)$. Then every weak solution $u$ to $(2.5)$ is a mild solution, that is, $u \in C([0, T] ; V) \cap L^{2}\left(0, T ; V_{1 / 2}\right) \cap C^{\hat{\beta}}\left([0, T] ; V_{-\delta}\right)$ and satisfies for every $t \in[0, T]$ the following integral formulation in $V$ :

$$
u(t)=S(t) u_{0}+\int_{0}^{t} S(t-r) B(u(r), u(r)) d r+\int_{0}^{t} S(t-r) G(u(r)) \omega^{\prime}(r) d r .
$$

Proof. Suppose that $u$ fulfills (4.3). Then

$$
t \mapsto B(u(t), u(t))+G(u(t)) \omega^{\prime}(t) \in L^{2}(0, T ; V)
$$

such that

$$
t \mapsto \int_{0}^{t} S(t-r) B(u(r), u(r)) d r+\int_{0}^{t} S(t-r) G(u(r)) \omega^{\prime}(r) d r \in C([0, T] ; V),
$$

see Pazy [25], proof of Theorem 4.3.1. In addition, every Galerkin-approximation solution of (5.1) satisfies

$$
\begin{aligned}
\left(u_{n}(t), \varphi\right)_{V} & =\left(S(t) P_{n} u_{n}(0), \varphi\right)_{V}+\int_{0}^{t}\left(S(t-r) P_{n} B\left(u_{n}(r), u_{n}(r)\right), \varphi\right)_{V} d r \\
& +\int_{0}^{t}\left(S(t-r) P_{n} G\left(u_{n}(r)\right) \omega^{\prime}(r), \varphi\right)_{V} d r
\end{aligned}
$$

for every $\varphi \in V$ and every $t \in[0, T]$. From the convergence of $\left(u_{n}\right)_{n \in \mathbb{N}}$ in $L^{2}(0, T ; V)$ and the boundedness in $L^{2}\left(0, T ; V_{1 / 2}\right)$ it follows that the right hand side of (5.3) converges to

$$
\left(S(t) u_{0}, \varphi\right)_{V}+\int_{0}^{t}(S(t-r) B(u(r), u(r)), \varphi)_{V} d r+\int_{0}^{t}\left(S(t-r) G(u(r)) \omega^{\prime}(r), \varphi\right)_{V} d r
$$

for every $t \in[0, T]$. On the other hand, from the proof of Proposition 5.1 we know that $\left(u_{n}\right)_{n \in \mathbb{N}}$ converges to $u$ in $C\left([0, T] ; V_{-\delta}\right)$ and hence $u_{n}(t)$ converges to $u(t)$ in $V_{-\delta}$ for every $t \in[0, T]$. Since the right hand side is in $V$ for every $t \in[0, T], u(t)$ is too. Also, following the same reasoning than in Proposition 5.1, one can prove that $\left(u_{n}\right)_{n \in \mathbb{N}}$ is bounded in $C^{\gamma}\left([0, T] ; V_{-\hat{\delta}}\right)$ for $\gamma=\hat{\beta}+\varepsilon$ and $\hat{\delta}=\delta-\varepsilon$ for small enough $\varepsilon>0$ such that $\hat{\delta}>\gamma$. Then it suffices to apply Theorem 2.2 (ii) to conclude the proof. 
From now on, we often use the following property, which is a consequence of the definition of Beta function: for every $0 \leq s<t \leq T, a, b>-1$,

$$
\int_{s}^{t}(r-s)^{a}(t-r)^{b} d r=c(t-s)^{a+b+1}
$$

where $c$ only depends on $a$ and $b$.

Next we develop a priori estimates that later we need to derive the existence of a solution for a general $\omega \in$ $C^{\beta^{\prime}}([0, T] ; V)$. We cannot use the estimate from Proposition 5.1 because the sequence $\left(\omega_{n}\right)_{n \in \mathbb{N}}$ approximating $\omega$ in $C^{\beta^{\prime}}([0, T] ; V)$ is not in general uniformly bounded in $L^{\infty}(0, T ; V)$. That is why in the following estimates the norm $\|\omega \mid\|_{\beta^{\prime}}$ appears.

Lemma 5.3. Assume that $1 / 2<\hat{\beta}<\beta^{\prime}, 1-\beta^{\prime}<\alpha<\hat{\beta}, \delta \in(\hat{\beta}, 1), u_{0} \in V$, $\omega$ is a piecewise linear continuous function and $G$ satisfies $(\mathbf{G})$. Then, if $u$ is a weak solution to (2.5) in the sense of Definition 4.3, there is a constant $c>0$ such that for $t \in[0, T]$

$$
\|u(t)\|^{2}+2 \int_{0}^{t}\|u(r)\|_{V_{1 / 2}}^{2} d r \leq\left\|u_{0}\right\|^{2}+c\|\| \omega\left\|_{\beta^{\prime}} t^{\beta^{\prime}}\right\| u\left\|_{C, 0, t}+c\right\|\|\omega\|\left\|_{\beta^{\prime}} t^{\hat{\beta}+\beta^{\prime}}\left(1+\|u\|_{C, 0, t}\right)\right\|\|u\| \|_{\hat{\beta},-\delta, 0, t} .
$$

Proof. Applying the formula of the square norm, see Teman [29] Lemma III.1.2, using the skew-symmetric property (2.4), and finally integrating over $(0, t)$, this gives us for every $t \in[0, T]$ the following energy inequality

$$
\|u(t)\|^{2}+2 \int_{0}^{t}\|u(r)\|_{V_{1 / 2}}^{2} d r \leq\left\|u_{0}\right\|^{2}+2\left|\int_{0}^{t}\left(G^{*}(u(r)) u(r), \omega^{\prime}(r)\right)_{V} d r\right| .
$$

The integral on the right hand side of the previous expression can be interpreted in the sense of Section 3 using fractional derivatives. Since for any $r$ we have $\left\|D_{0+}^{\alpha} G^{*}(u(r)) u(r)\right\|<\infty$ the expression $D_{0+}^{\alpha} G^{*}(u(r)) u(r)$ is an element in the space of Hilbert-Schmidt-operators $L_{2}(V, \mathbb{R}) \simeq V$. Moreover, from the definition of the fractional derivative it is easy to derive that

$$
\left\|D_{t-}^{1-\alpha} \omega_{t-}[r]\right\| \leq c\|\| \omega \|_{\beta^{\prime}}(t-r)^{\alpha+\beta^{\prime}-1}
$$

and therefore we get

$$
\begin{gathered}
\left|\int_{0}^{t}\left(G^{*}(u(r)) u(r), \omega^{\prime}(r)\right)_{V} d r\right| \leq c \mid\|\omega\|_{\beta^{\prime}} \int_{0}^{t}(t-r)^{\alpha+\beta^{\prime}-1}\left(\frac{\left\|G^{*}(u(r)) u(r)\right\|}{r^{\alpha}}\right. \\
\left.+\int_{0}^{r} \frac{\left\|G^{*}(u(r)) u(r)-G^{*}(u(q)) u(q)\right\|}{(r-q)^{1+\alpha}} d q\right) d r .
\end{gathered}
$$

Trivially the boundedness of $G$ implies that $\left\|G^{*}(u) u\right\| \leq c_{G}\|u\|$ for $u \in V$ and therefore

$$
\frac{\left\|G^{*}(u(r)) u(r)\right\|}{r^{\alpha}} \leq \frac{c_{G}\|u\|_{C, 0, t}}{r^{\alpha}}, \quad r \in[0, t] .
$$

The boundedness and the Lipschitz-continuity of $G$ imply

$$
\begin{aligned}
\int_{0}^{r} & \frac{\left\|G^{*}(u(r)) u(r)-G^{*}(u(q)) u(q)\right\|}{(r-q)^{1+\alpha}} d q \\
& \leq c_{G} \int_{0}^{r} \frac{\|u(r)-u(q)\|_{V_{-\delta}}}{(r-q)^{1+\alpha}} d q+\|u\|_{C, 0, t} \int_{0}^{r} \frac{\left\|G^{*}(u(r))-G^{*}(u(q))\right\|_{L_{2}\left(V_{-\delta}, V\right)}}{(r-q)^{1+\alpha}} d q \\
& \leq\left(c_{G}+c_{D G}\|u\|_{C, 0, t}\right) \mid\|u\| \|_{\hat{\beta},-\delta, 0, t} \int_{0}^{r}(r-q)^{-1-\alpha+\hat{\beta}} d q \\
& =c\left(c_{G}+c_{D G}\|u\|_{C, 0, t}\right)\|\| u \|_{\hat{\beta},-\delta, 0, t} r^{\hat{\beta}-\alpha} .
\end{aligned}
$$

Hence, for an appropriate $c>0$

$$
\left|\int_{0}^{t}\left(G^{*}(u(r)) u(r), \omega^{\prime}(r)\right)_{V} d r\right| \leq c\left|\|\omega\|_{\beta^{\prime}} t^{\beta^{\prime}}\|u\|_{C, 0, t}+c\right|\|\omega\|\left\|_{\beta^{\prime}} t^{\hat{\beta}+\beta^{\prime}}\left(1+\|u\|_{C, 0, t}\right) \mid\right\| u\|\|_{\hat{\beta},-\delta, 0, t} .
$$


Lemma 5.4. Under the same conditions of Lemma 5.3, if $u$ is a solution to (2.5) there exist constants $c, \bar{c}>0$ such that for $t \in[0, T]$

$$
\|\| u\left|\left\|_{\hat{\beta},-\delta, 0, t} \leq \bar{c} t^{\delta-\hat{\beta}}\right\| u_{0}\left\|+c\left(t^{1-\hat{\beta}}+t^{\delta+1 / 2-\hat{\beta}}\right)\right\| u\left\|_{C, 0, t}^{2}+c \mid\right\| \omega\|\|_{\beta^{\prime}} t^{\beta^{\prime}-\hat{\beta}}\left(1+t^{\hat{\beta}}\|\mid u\| \|_{\hat{\beta},-\delta, 0, t}\right) .\right.
$$

Proof. Consider (5.2) written as

$$
u(t)=S(t) u_{0}+A^{1 / 2} \int_{0}^{t} S(t-r) A^{-1 / 2} B(u(r), u(r)) d r+\int_{0}^{t} S(t-r) G(u(r)) \omega^{\prime}(r) d r .
$$

Then the following splitting is considered:

$$
\begin{aligned}
& A^{-\delta}(u(q)-u(p))=A^{-\delta}(S(q)-S(p)) u_{0}+A^{-\delta+1 / 2} \int_{p}^{q} S(q-r) A^{-1 / 2} B(u(r), u(r)) d r \\
& +A^{-\delta+1 / 2} \int_{0}^{p}(S(q-r)-S(p-r)) A^{-1 / 2} B(u(r), u(r)) d r \\
& +A^{-\delta} \int_{p}^{q} S(q-r) G(u(r)) \omega^{\prime}(r) d r+A^{-\delta} \int_{0}^{p}(S(q-r)-S(p-r)) G(u(r)) \omega^{\prime}(r) d r \\
& =: I_{1}+I_{2}+I_{3}+I_{4}+I_{5} .
\end{aligned}
$$

For the term related to the initial condition, due to the fact that $\delta \in(\hat{\beta}, 1)$ and $(2.1),(2.2)$ we have

$$
\sup _{0 \leq p<q \leq t} \frac{\left\|I_{1}\right\|}{(q-p)^{\hat{\beta}}} \leq \sup _{0 \leq p<q \leq t} \frac{\left\|A^{-\delta}(S(q-p)-\mathrm{Id}) S(p) u_{0}\right\|}{(q-p)^{\hat{\beta}}} \leq \bar{c} \sup _{0 \leq p<q \leq t} \frac{(q-p)^{\delta}\left\|u_{0}\right\|}{(q-p)^{\hat{\beta}}} \leq \bar{c} t^{\delta-\hat{\beta}}\left\|u_{0}\right\| .
$$

Moreover, due to Lemma 2.1 and taking into account that $V \subset V_{-\delta+1 / 2}$,

$$
\begin{aligned}
\sup _{0 \leq p<q \leq t} \frac{\left\|I_{2}\right\|}{(q-p)^{\hat{\beta}}} & \leq \sup _{0 \leq p<q \leq t} \frac{1}{(q-p)^{\hat{\beta}}} \int_{p}^{q}\left\|A^{-\delta+1 / 2} S(q-r) A^{-1 / 2} B(u(r), u(r))\right\| d r \\
& \leq \sup _{0 \leq p<q \leq t} \frac{c}{(q-p)^{\hat{\beta}}} \int_{p}^{q}\left\|A^{-1 / 2} B(u(r), u(r))\right\| d r \\
& \leq \sup _{0 \leq p<q \leq t} \frac{c}{(q-p)^{\hat{\beta}}}(q-p)\|u\|_{C, 0, t}^{2} \leq c t^{1-\hat{\beta}}\|u\|_{C, 0, t}^{2} .
\end{aligned}
$$

For $I_{3}$, thanks to Lemma 2.1 and $(2.3)$,

$$
\begin{aligned}
\sup _{0 \leq p<q \leq t} \frac{\left\|I_{3}\right\|}{(q-p)^{\hat{\beta}}} & \leq \sup _{0 \leq p<q \leq t} \frac{c}{(q-p)^{\hat{\beta}}} \int_{0}^{p}\left\|A^{-\delta+1 / 2}(S(q-p)-\mathrm{Id}) S(p-r) A^{-1 / 2} B(u(r), u(r))\right\| d r \\
& \leq c\|u\|_{C, 0, t}^{2} \sup _{0 \leq p \leq t} \int_{0}^{p}(p-r)^{\delta-1 / 2-\hat{\beta}} d r \\
& \leq c t^{\delta+1 / 2-\hat{\beta}}\|u\|_{C, 0, t}^{2} .
\end{aligned}
$$

Similar estimates to those of $I_{4}, I_{5}$ can be found in [6]. However, and for the completeness of the presentation, we also show these technical estimates in this paper, but we have shifted these calculations into the Appendix Section, see Lemma 8.1(i) and Corollary 8.2 (i) below, where it is proven that

$$
\sup _{0 \leq p<q \leq t} \frac{\left\|I_{4}\right\|+\left\|I_{5}\right\|}{(q-p)^{\hat{\beta}}} \leq c t^{\beta^{\prime}-\hat{\beta}}\||\omega|\|_{\beta^{\prime}}\left(1+t^{\hat{\beta}}\|u\| \|_{\hat{\beta},-\delta, 0, t}\right) .
$$

Hence, collecting all the estimates for the expressions $I_{j}$ the inequality (5.7) is obtained.

Lemma 5.5. Under the assumptions of Lemma 5.3, if $u_{n}$ is a solution of (4.3) on $[0, T]$ with initial condition $u_{0} \in V$ and driven by a piecewise linear continuous path $\omega_{n}$ where $\left(\omega_{n}\right)_{n \in \mathbb{N}}$ is bounded in $C^{\beta^{\prime}}([0, T] ; V)$, then $\left(u_{n}\right)_{n \in \mathbb{N}}$ is uniformly bounded in $C^{\hat{\beta}}\left([0, T] ; V_{-\delta}\right) \cap C([0, T] ; V)$.

The proof of the previous result rests upon the technical Lemmas 8.3-8.5 whose proofs are presented into the Appendix section. 
Remark 5.6. We emphasize that we consider Hölder-continuity with respect to the space $V_{-\delta}$, although all the estimates of the Hölder-norms also make sense for smaller $\delta$. However, the initial condition $u_{0}$ is the responsible of having to consider $\delta \in(\hat{\beta}, 1)$, since in (5.7) the exponent in the term $t^{\delta-\hat{\beta}}$ multiplying $\left\|u_{0}\right\|$ must be positive.

\section{Construction of solutions}

We are now able to construct solutions for the stochastic equation (2.5) and give the main result of this paper. We consider a sequence of solutions $\left(u_{n}\right)_{n \in \mathbb{N}}$ to $(2.5)$ driven by $\left(\omega_{n}\right)_{n \in \mathbb{N}}$, a sequence of piecewise linear continuous approximations of $\omega$ converging to $\omega$ where $\omega$ satisfies Remark 3.2.

First we formulate a general uniqueness theorem.

Theorem 6.1. Suppose that there are two mild solutions $u_{1}$, $u_{2}$ of $(4.2)$ with $u_{1}(0)=u_{2}(0)=u_{0} \in V$ and driven by the same path $\omega$. Then, under the given assumptions on $A, B$ and $G$ we have $u_{1}(t)=u_{2}(t)$ for $t \in[0, T]$.

Proof. Assume that there exists a maximal interval $\left[0, t_{0}\right]$ contained in $[0, T]$ such that $\Delta u:=u_{1}-u_{2}$ is zero on this interval being $t_{0}<T$. Then there exists a $0<\mu<1$ such that $\Delta u \neq 0$ on $\left(t_{0}, t_{0}+\mu\right]$.

We divide the proof in several steps:

(i) First we want to estimate

$$
\||| \Delta u \mid\|_{\beta,-\delta, t_{0}, t_{0}+\mu}=\sup _{t_{0} \leq s<t \leq t_{0}+\mu} \frac{\|\Delta u(t)-\Delta u(s)\|_{V_{-\delta}}}{(t-s)^{\beta}} .
$$

Regarding the non-stochastic integral, we have to estimate

$$
\begin{aligned}
\frac{1}{(t-s)^{\beta}} & \left\|\int_{s}^{t} S(t-r) A^{-\delta}\left(B\left(u_{1}(r), u_{1}(r)\right)-B\left(u_{2}(r), u_{2}(r)\right)\right) d r\right\| \\
& +\frac{1}{(t-s)^{\beta}}\left\|\int_{t_{0}}^{s}(S(t-r)-S(s-r)) A^{-\delta}\left(B\left(u_{1}(r), u_{1}(r)\right)-B\left(u_{2}(r), u_{2}(r)\right)\right) d r\right\| \\
& =: J_{1}+J_{2} .
\end{aligned}
$$

Since $V_{-1 / 2} \subset V_{-\delta}$, from Lemma 2.1 we obtain

$$
\begin{aligned}
\left\|A^{-\delta}\left(B\left(u_{1}(r), u_{1}(r)\right)-B\left(u_{2}(r), u_{2}(r)\right)\right)\right\| & \leq c\left\|B\left(\Delta u(r), u_{1}(r)\right)\right\|_{V_{-1 / 2}}+c\left\|B\left(u_{2}(r), \Delta u(r)\right)\right\|_{V_{-1 / 2}} \\
& \leq c\|\Delta u(r)\|\left(\left\|u_{1}(r)\right\|+\left\|u_{2}(r)\right\|\right) .
\end{aligned}
$$

Therefore

$$
J_{1} \leq \frac{c}{(t-s)^{\beta}} \int_{s}^{t}\|\Delta u(r)\|\left(\left\|u_{1}(r)\right\|+\left\|u_{2}(r)\right\|\right) d r \leq c \mu^{1-\beta}\|\Delta u\|_{C, t_{0}, t_{0}+\mu}\left(\left\|u_{1}\right\|_{C, t_{0}, t_{0}+\mu}+\left\|u_{2}\right\|_{C, t_{0}, t_{0}+\mu}\right) .
$$

Notice also that using the properties of the semigroup $S$

and thus

$$
\begin{aligned}
& \left\|(S(t-r)-S(s-r)) A^{-\delta}\left(B\left(u_{1}(r), u_{1}(r)\right)-B\left(u_{2}(r), u_{2}(r)\right)\right)\right\| \\
& =\left\|(S(t-s)-\mathrm{id}) S(s-r)\left(B\left(u_{1}(r), u_{1}(r)\right)-B\left(u_{2}(r), u_{2}(r)\right)\right)\right\|_{V_{-\delta}} \\
& \quad \leq c(t-s)^{\delta}\left\|\left(B\left(u_{1}(r), u_{1}(r)\right)-B\left(u_{2}(r), u_{2}(r)\right)\right)\right\| \\
& \quad \leq c(t-s)^{\delta}\|\Delta u(r)\|\left(\left\|u_{1}(r)\right\|_{V_{1 / 2}}+\left\|u_{2}(r)\right\|_{V_{1 / 2}}\right),
\end{aligned}
$$

$$
\begin{aligned}
J_{2} & \leq \frac{1}{(t-s)^{\beta}} \int_{t_{0}}^{s}(t-s)^{\delta}\|\Delta u(r)\|\left(\left\|u_{1}(r)\right\|_{V_{1 / 2}}+\left\|u_{2}(r)\right\|_{V_{1 / 2}}\right) d r \\
& \leq \mu^{\frac{1}{2}+\delta-\beta}\|\Delta u\|_{C, t_{0}, t_{0}+\mu}\left(\left\|u_{1}\right\|_{L^{2}\left(0, T, V_{1 / 2}\right)}+\left\|u_{2}\right\|_{L^{2}\left(0, T, V_{1 / 2}\right)}\right) .
\end{aligned}
$$

To analyze the terms corresponding to the stochastic integral, that is,

$$
\sup _{t_{0} \leq s<t \leq t_{0}+\mu} \frac{\left\|\int_{s}^{t} S(t-r)\left(G\left(u_{1}(r)\right)-G\left(u_{2}(r)\right)\right) d \omega-\int_{t_{0}}^{s}(S(t-r)-S(s-r))\left(G\left(u_{1}(r)\right)-G\left(u_{2}(r)\right)\right) d \omega\right\|_{V_{-\delta}}}{(t-s)^{\beta}}
$$


we can consider the estimates of $I_{4}, I_{5}$ given in the Appendix, replacing $\| A^{-\delta}\left(G(u(r)) \|_{L_{2}(V)}\right.$ by

$$
\left\|A^{-\delta}\left(G\left(u_{1}(r)\right)-G\left(u_{2}(r)\right)\right)\right\|_{L_{2}(V)} \leq c_{D G}\|\Delta u(r)\|_{V_{-\delta}}
$$

and $\left\|A^{-\delta}(G(u(r))-G(u(q)))\right\|_{L_{2}(V)}$ by

$$
\begin{aligned}
\| A^{-\delta}\left(G\left(u_{1}(r)\right)\right. & \left.-G\left(u_{2}(r)\right)-\left(G\left(u_{1}(q)\right)-G\left(u_{2}(q)\right)\right)\right) \|_{L_{2}(V)} \\
& \leq c_{D G}\|\Delta u(r)-\Delta u(q)\|_{V_{-\delta}}+c_{D^{2} G}\left(\| \Delta u ( r ) \| _ { V _ { - \delta } } \left(\left\|u_{1}(r)-u_{1}(q)\right\|_{V_{-\delta}}\right.\right. \\
& \left.+\left\|u_{2}(r)-u_{2}(q)\right\|_{V_{-\delta}}\right),
\end{aligned}
$$

where these two above estimates follow by $(\mathbf{G})$. Then following the steps of Lemma 8.1 and taking into account

$$
\|\Delta u(r)\|_{V_{-\delta}}=\left\|\Delta u(r)-\Delta u\left(t_{0}\right)\right\|_{V_{-\delta}} \leq\|\| \Delta u\|\|_{\beta,-\delta, t_{0}, t_{0}+\mu}\left(r-t_{0}\right)^{\beta},
$$

which is true due to the fact that $\Delta u\left(t_{0}\right)=0$, we obtain the following term as an upper bound of the stochastic part:

$$
c|||\omega|||_{\beta^{\prime}} \mu^{\beta^{\prime}}|||\Delta u|\left\|\left.\right|_{\beta,-\delta, t_{0}, t_{0}+\mu}+c\left|\left\|\left.\omega||\right|_{\beta^{\prime}} \mu^{\beta^{\prime}}\left(\left|\left\|\left.u_{1}||\right|_{\beta,-\delta, 0, T}+\right\|\right|\left|u_{2}\right| \|\left.\right|_{\beta,-\delta, 0, T}\right)\right\| \Delta u \|_{C, t_{0}, t_{0}+\mu} .\right.\right.
$$

Collecting everything we get

$$
\left|\left\|\Delta u||_{\beta,-\delta, t_{0}, t_{0}+\mu} \leq c_{\mu}^{1}\right\|\right| \Delta u \mid\left\|_{\beta,-\delta, t_{0}, t_{0}+\mu}+c_{\mu}^{2}\right\| \Delta u \|_{C, t_{0}, t_{0}+\mu},
$$

with

$$
\begin{aligned}
c_{\mu}^{1}= & c \mu^{\beta^{\prime}}|\|\omega\||_{\beta^{\prime}}, \\
c_{\mu}^{2}= & c\left(\mu^{\frac{1}{2}+\delta-\beta}\left(\left\|u_{1}\right\|_{L^{2}\left(0, T, V_{1 / 2}\right)}+\left\|u_{2}\right\|_{L^{2}\left(0, T, V_{1 / 2}\right)}\right)+\mu^{\beta^{\prime}}\||| \mid\|_{\beta^{\prime}}\left(\left|\left\|u_{1}\left|\left\|_{\beta,-\delta, 0, T}+\right\|\right|\left|u_{2}\right|\right\|\right|_{\beta,-\delta, 0, T}\right)\right. \\
& \left.+\mu^{1-\beta}\left(\left\|u_{1}\right\|_{C, t_{0}, t_{0}+\mu}+\left\|u_{2}\right\|_{C, t_{0}, t_{0}+\mu}\right)\right) .
\end{aligned}
$$

(ii) In this second step we are interested in estimating $\|\Delta u\|_{C, t_{0}, t_{0}+\mu}$. The non-stochastic part gives us

$$
\begin{aligned}
& \sup _{t_{0} \leq t \leq t_{0}+\mu}\left\|\int_{t_{0}}^{t} S(t-r)\left(B\left(u_{1}(r), u_{1}(r)\right)-B\left(u_{2}(r), u_{2}(r)\right)\right) d r\right\| \\
\leq & c \sup _{t_{0} \leq t \leq t_{0}+\mu} \int_{t_{0}}^{t}\|\Delta u(r)\|\left(\left\|u_{1}(r)\right\|_{V_{1 / 2}}+\left\|u_{2}(r)\right\|_{V_{1 / 2}}\right) d r \\
\leq & c \mu^{\frac{1}{2}}\left(\left\|u_{1}\right\|_{L^{2}\left(0, T, V_{1 / 2}\right)}+\left\|u_{2}\right\|_{L^{2}\left(0, T, V_{1 / 2}\right)}\right)\|\Delta u\|_{C, t_{0}, t_{0}+\mu} .
\end{aligned}
$$

To study the norm of the stochastic integral, for $t \in\left[t_{0}, t_{0}+\mu\right]$ we split it as follows

$$
\begin{aligned}
\|\mid \omega\| \|_{\beta^{\prime}} \int_{t_{0}}^{t}(t- & r)^{\alpha+\beta^{\prime}-1}\left(\frac{\left\|S(t-r)\left(G\left(u_{1}(r)\right)-G\left(u_{2}(r)\right)\right)\right\|_{L_{2}(V)}}{\left(r-t_{0}\right)^{\alpha}}\right. \\
& +\int_{t_{0}}^{r} \frac{\left\|(S(t-r)-S(t-\hat{r}))\left(G\left(u_{1}(r)\right)-G\left(u_{2}(r)\right)\right)\right\|_{L_{2}(V)}}{(r-\hat{r})^{\alpha+1}} d \hat{r} \\
& \left.+\int_{t_{0}}^{r} \frac{\left\|S(t-\hat{r})\left(\left(G\left(u_{1}(r)\right)-G\left(u_{2}(r)\right)\right)-\left(G\left(u_{1}(\hat{r})\right)-G\left(u_{2}(\hat{r})\right)\right)\right)\right\|_{L_{2}(V)}}{(r-\hat{r})^{\alpha+1}} d \hat{r}\right) d r \\
=: J_{3}(t) & +J_{4}(t)+J_{5}(t) .
\end{aligned}
$$

Following the steps of Lemma 8.1, thanks to (G) we obtain

$$
\begin{aligned}
& \sup _{t_{0} \leq t \leq t_{0}+\mu} J_{3}(t) \leq c \mid\|\omega\|\left\|_{\beta^{\prime}} \mu^{\beta^{\prime}}\right\| \Delta u \|_{C, t_{0}, t_{0}+\mu}, \\
& \sup _{t_{0} \leq t \leq t_{0}+\mu} J_{4}(t) \leq c \mid\|\omega\|\left\|_{\beta^{\prime}} \mu^{\beta^{\prime}}\right\| \Delta u \|_{C, t_{0}, t_{0}+\mu} .
\end{aligned}
$$


Finally, using again $(\mathbf{G})$, since $\|\Delta u(r)\|_{V_{-\delta}} \leq c\|\Delta u(r)\|$,

$$
\begin{aligned}
& \sup _{t_{0} \leq t \leq t_{0}+\mu} J_{5}(t) \leq c\left|\|\omega \mid\|_{\beta^{\prime}} \int_{t_{0}}^{t}(t-r)^{\alpha+\beta^{\prime}-1}\right. \\
& \times\left(\int_{t_{0}}^{r} \frac{\|\Delta u(r)-\Delta u(\hat{r})\|_{V_{-\delta}}+\|\Delta u(r)\|\left(\left\|u_{1}(r)-u_{1}(\hat{r})\right\|_{V_{-\delta}}+\left\|u_{2}(r)-u_{2}(\hat{r})\right\|_{V_{-\delta}}\right)}{(r-\hat{r})^{\alpha+1}} d \hat{r}\right) d r \\
& \leq c|||\omega|||_{\beta^{\prime}}\left(| | \left|\Delta u\left\|\left.\right|_{\beta,-\delta, t_{0}, t_{0}+\mu}+\right\| \Delta u \|_{C, t_{0}, t_{0}+\mu}\left(||\left|u_{1}\right|||_{\beta,-\delta, t_{0}, t_{0}+\mu}+||\left|u_{2}\right| \mid \|_{\beta,-\delta, t_{0}, t_{0}+\mu}\right)\right.\right. \\
& \times \sup _{t_{0} \leq t \leq t_{0}+\mu} \int_{t_{0}}^{t}(t-r)^{\alpha+\beta^{\prime}-1}\left(\int_{t_{0}}^{r}(r-\hat{r})^{\beta-\alpha-1} d \hat{r}\right) d r \\
& \leq c|||\omega| \mid \|_{\beta^{\prime}} \mu^{\beta+\beta^{\prime}}\left(|||\Delta u|\left\|_{\beta,-\delta, t_{0}, t_{0}+\mu}+\right\| \Delta u \|_{C, t_{0}, t_{0}+\mu}\left(||\left|u_{1}\right|\left|\left\|_{\beta,-\delta, 0, T}+||\left|u_{2}\right|\right\| \|_{\beta,-\delta, 0, T}\right)\right) .\right.
\end{aligned}
$$

Hence,

$$
\|\Delta u\|_{C, t_{0}, t_{0}+\mu} \leq c_{\mu}^{3}\|\Delta u\|_{C, t_{0}, t_{0}+\mu}+c_{\mu}^{4}\|\| \Delta u\|\|_{\beta,-\delta, t_{0}, t_{0}+\mu},
$$

with

$$
\begin{aligned}
c_{\mu}^{3}= & c\left(\mu^{\frac{1}{2}}\left(\left\|u_{1}\right\|_{L^{2}\left(0, T, V_{1 / 2}\right)}+\left\|u_{2}\right\|_{L^{2}\left(0, T, V_{1 / 2}\right)}\right)+\mu^{\beta^{\prime}}\||\omega|\|_{\beta^{\prime}}\right. \\
& +\mu^{\beta^{\prime}+\beta}|||\omega| \|_{\beta^{\prime}}\left(\left\|u_{1}\left|\left\|_{\beta,-\delta, 0, T}+\right\| u_{2} \|\right|_{\beta,-\delta, 0, T}\right)\right), \\
c_{\mu}^{4}= & c \mu^{\beta^{\prime}+\beta}|||\omega| \|_{\beta^{\prime}} .
\end{aligned}
$$

Therefore, solving the system given by (6.3) and (6.5) means that we have to solve a system of inequalities, namely

$$
X \leq c_{\mu}^{1} X+c_{\mu}^{2} Y, \quad Y \leq c_{\mu}^{3} Y+c_{\mu}^{4} X
$$

with $c_{\mu}^{i}$ given by (6.4) and (6.6). It is now straightforward to check that for a small enough $\mu \in(0,1)$ we obtain that $\|\Delta u\|_{C, t_{0}, t_{0}+\mu}=0$, which contradicts the fact that the maximal interval of uniqueness is $\left[0, t_{0}\right]$. Hence the solution of (2.5) is unique.

Finally, we can prove the main theorem of the paper:

Theorem 6.2. Under the assumptions of Lemma 5.3 there exists a mild solution to the stochastic shell-model (2.5) with driving function $\omega \in C^{\beta^{\prime}}([0, T] ; V)$.

Proof. We divide the proof in several steps:

(i) Let $\left(\omega_{n}\right)_{n \in \mathbb{N}}$ be a sequence of piecewise linear continuous functions converging to $\omega$ in $C^{\beta^{\prime}}([0, T] ; V)$, see Remark 3.2, and let $\left(u_{n}\right)_{n \in \mathbb{N}}$ be the sequence of unique solutions driven by $\left(\omega_{n}\right)_{n \in \mathbb{N}}$ with initial condition $u_{0} \in V$. From Lemma 5.5 we know that $\left(u_{n}\right)_{n \in \mathbb{N}}$ is uniformly bounded in $C^{\hat{\beta}}\left([0, T] ; V_{-\delta}\right) \cap C([0, T] ; V)$. Then (5.5) implies that $\left(\left\|u_{n}\right\|_{L^{2}\left(0, T ; V_{1 / 2}\right)}\right)_{n \in \mathbb{N}}$ is also bounded and hence $\left(u_{n}\right)_{n \in \mathbb{N}}$ is relatively weak compact in $L^{2}\left(0, T ; V_{1 / 2}\right)$. Furthermore, this sequence is relatively compact in $L^{2}(0, T ; V) \cap C\left([0, T] ; V_{-\delta}\right)$ by Theorem 2.2 (i).

Moreover, thanks to Corollary 8.2 (ii), for $\hat{\delta}=\delta-\varepsilon$ with $\varepsilon>0$ arbitrarily small, we have

$$
\left\|| | u _ { n } \left|\| _ { \hat { \beta } , - \hat { \delta } , 0 , t } \leq c t ^ { \hat { \delta } - \hat { \beta } } \| u _ { n } ( 0 ) \| + c ( t ^ { 1 - \hat { \beta } } + t ^ { \hat { \delta } - 1 / 2 - \hat { \beta } } ) \| u _ { n } \left\|_{C, 0, t}^{2}+c\left|\left\|\omega_{n}\right\|\right|_{\beta^{\prime}} t^{\beta^{\prime}-\hat{\beta}-\varepsilon}\left(1+t^{\hat{\beta}}\left\|\left|u_{n}\right|\right\|_{\hat{\beta},-\hat{\delta}, 0, t}\right) .\right.\right.\right.
$$

Following a similar reasoning to the one of Lemma 8.5, we can make small the coefficient in front of ||$\left|u_{n}\right|||_{\hat{\beta},-\hat{\delta}, 0, t}$ on the left hand side of (6.7) such that we can solve such an inequality. Moreover, since $\left(\omega_{n}\right)_{n \in \mathbb{N}}$ converges to $\omega$, we obtain that $\left(u_{n}\right)_{n \in \mathbb{N}}$ is uniformly bounded in $C^{\hat{\beta}}\left([0, T] ; V_{-\hat{\delta}}\right)$. Hence, by Theorem 2.2 (ii), this sequence in relatively compact in $C^{\beta}\left([0, T] ; V_{-\delta}\right)$ with $\beta<\hat{\beta}$.

(ii) Let $\left(u_{n^{\prime}}\right)_{n^{\prime} \in \mathbb{N}}$ be a subsequence converging to some limit point $u \in L^{2}\left(0, T ; V_{1 / 2}\right) \cap C^{\beta}\left([0, T] ; V_{-\delta}\right)$. Let us denote this subsequence simply by $\left(u_{n}\right)_{n \in \mathbb{N}}$. Then, since $B: V_{\frac{1}{2}} \times V_{-\delta} \rightarrow V_{-\delta}$ and also $B: V_{-\delta} \times V_{\frac{1}{2}} \rightarrow V_{-\delta}$ 
and $u_{n}(0)-u(0)=0$, applying Lemma 2.1 we have

$$
\begin{aligned}
& \left\|\int_{0}^{t} S(t-r)\left(B\left(u_{n}(r), u_{n}(r)\right)-B(u(r), u(r))\right) d r\right\|_{V_{-\delta}} \\
& \leq \int_{0}^{t}\left(\left\|B\left(u_{n}(r), u_{n}(r)\right)-B\left(u(r), u_{n}(r)\right)\right\|_{V_{-\delta}}+\left\|B\left(u(r), u_{n}(r)\right)-B(u(r), u(r))\right\|_{V_{-\delta}}\right) d r \\
& \leq c \int_{0}^{t}\left(\left\|u_{n}(r)\right\|_{V_{1 / 2}}+\|u(r)\|_{V_{1 / 2}}\right)\left\|u(r)-u_{n}(r)\right\|_{V_{-\delta}} d r \\
& \leq c\left\|u-u_{n} \mid\right\|_{\beta,-\delta, 0, T} \int_{0}^{t} r^{\beta}\left(\left\|u_{n}(r)\right\|_{V_{1 / 2}}+\|u(r)\|_{V_{1 / 2}}\right) d r \\
& \leq c T^{\beta+\frac{1}{2}}\|\| u-u_{n}\|\|_{\beta,-\delta, 0, T}\left(\left\|u_{n}\right\|_{L^{2}\left(0, T ; V_{1 / 2}\right)}+\|u\|_{L^{2}\left(0, T ; V_{1 / 2}\right)}\right)
\end{aligned}
$$

which shows the convergence in $V_{-\delta}$ of the left hand side to zero.

For the stochastic integral we consider the splitting

$$
\begin{aligned}
& \left\|\int_{0}^{t} S(t-r) G\left(u_{n}(r)\right) d \omega_{n}(r)-\int_{0}^{t} S(t-r) G(u(r)) d \omega(r)\right\|_{V_{-\delta}} \\
& \quad \leq\left\|\int_{0}^{t} S(t-r) G\left(u_{n}(r)\right) d\left(\omega_{n}(r)-\omega(r)\right)\right\|_{V_{-\delta}}+\left\|\int_{0}^{t} S(t-r)\left(G\left(u_{n}(r)\right)-G(u(r))\right) d \omega_{n}(r)\right\|_{V_{-\delta}} .
\end{aligned}
$$

Similar to (5.12), an upper bound for the first integral on the right hand side is given by

$$
C T^{\beta^{\prime}}|| \omega_{n}-\omega \mid \|_{\beta^{\prime}}\left(1+T^{\beta}||\left|u_{n}\right| \|_{\beta,-\delta, 0, T}\right)
$$

and since the set $\left\{||\left|u_{n}\right| \|_{\beta,-\delta, 0, T}\right\}_{n \in \mathbb{N}}$ is bounded, we obtain the convergence in $V_{-\delta}$ of the first integral on the right hand side. Now using (6.1)-(6.2), setting $u_{1}=u_{n}, u_{2}=u$ we arrive at

$$
\begin{aligned}
\left\|\int_{0}^{t} S(t-r)\left(G\left(u_{n}(r)\right)-G(u(r))\right) d \omega_{n}(r)\right\|_{V_{-\delta}} & \leq c\left|\left\|\omega_{n}\left|\left\|\left.\right|_{\beta^{\prime}} T^{\beta^{\prime}}\right\|\right| u_{n}-u\right\| \|_{\beta,-\delta, 0, T}\right. \\
& \times\left(1+T^{\beta}\left(1+\left\|\left|\left\|u_{n}\left|\left\|\left.\right|_{\beta,-\delta, 0, T}+\right\|\|u\|\right|_{\beta,-\delta, 0, T}\right)\right)\right.\right.\right.
\end{aligned}
$$

which shows the convergence in $V_{-\delta}$ of the second integral.

Also, since $\left(u_{n}\right)_{n \in \mathbb{N}}$ converges to $u$ in $C\left([0, T] ; V_{-\delta}\right)$, for every $t \in[0, T]$ we have that $u_{n}(t) \rightarrow u(t)$ in $V_{-\delta}$.

(iii) Since $u \in L^{2}\left(0, T ; V_{1 / 2}\right) \cap L^{\infty}(0, T ; V)$ we have that $t \mapsto B(u(t), u(t)) \in L^{2}(0, T ; V)$ and hence the continuity in $V$ of the first integral of (4.2) with respect to $t$ follows. Moreover, since $u \in C^{\beta}\left([0, T] ; V_{-\delta}\right)$ by (G) we obtain that

$$
t \mapsto \int_{0}^{t} S(t-r) G(u(r)) d \omega \in C([0, T] ; V) .
$$

(iv) Collecting the above properties, on the one hand (i)-(ii) mean that $u \in C^{\beta}\left([0, T] ; V_{-\delta}\right) \cap L^{2}\left(0, T ; V_{1 / 2}\right)$ and $u$ satisfies (4.2) in $V_{-\delta}$. On the other hand, (iii) means that the right hand side of (4.2) belongs to $C([0, T] ; V)$, and hence also the left hand side. In conclusion, we have proven the existence of a mild solution $u$ to the stochastic shell-model in the sense of Definition 4.1.

\section{AN EXAMPLE OF DIFFUSION TERM}

We define the operator $G$ by a sequence of functions $g_{m}^{n}(u) \in \mathbb{C}$ with $u \in V_{-\delta}$, such that for $v \in V$ :

$$
(G(u) v)_{n}:=\sum_{n, m=1}^{\infty} g_{m}^{n}(u) v_{m} .
$$

We now define properties for this sequence such that $G$ satisfies the hypotheses $(\mathbf{G})$. For every $n, m=1, \ldots$, assume that

$$
\sup _{u \in V_{-\delta}} \sum_{n, m=1}^{\infty}\left|g_{m}^{n}(u)\right|^{2}=: c_{G}^{2}<\infty .
$$


In addition, let us assume that the functions $g_{m}^{n}$ are twice differentiable having the following properties: For $u, h \in V_{-\delta}$ and $\left(f_{k}\right)_{k \in \mathbb{N}}$ an orthonormal base in $V_{-\delta}$ we have that

$$
\begin{aligned}
& \sum_{n, m=1}^{\infty}\left(g_{m}^{n}(u+h)-g_{m}^{n}(u)-D g_{m}^{n}(u) h\right)^{2}=\sum_{n, m=1}^{\infty}\left(o_{u}^{n, m}\left(\|h\|_{V_{-\delta}}\right)\right)^{2}=o_{u}\left(\|h\|_{V_{-\delta}}\right)^{2}, \\
& \sup _{u \in V_{-\delta}} \sum_{n, m, k=1}^{\infty}\left|D g_{m}^{n}(u) f_{k}\right|^{2}=: c_{D G}^{2}<\infty .
\end{aligned}
$$

The $o_{u}^{n, m}, o_{u}$ have the usual properties: $\lim _{h \rightarrow 0}\left|o_{u}^{n, m}\left(\|h\|_{V_{-\delta}}\right)\right| /\|h\|_{V_{-\delta}}=0$ and similar for $o_{u}$. In addition we assume that for $u, h_{1}, h_{2} \in V_{-\delta}$

$$
\begin{aligned}
& \sum_{n, m=1}^{\infty}\left(D g_{m}^{n}\left(u+h_{2}\right) h_{1}-D g_{m}^{n}(u) h_{1}-D^{2} g_{m}^{n}(u) h_{1} h_{2}\right)^{2}=\sum_{n, m=1}^{\infty}\left(o_{u, h_{1}}^{n, m}\left(\left\|h_{2}\right\|_{V_{-\delta}}\right)\right)^{2}=:\left(o_{u, h_{1}}\left(\left\|h_{2}\right\|_{V_{-\delta}}\right)\right)^{2} \\
& \sup _{u \in V_{-\delta}} \sum_{n, m, k, l=1}^{\infty}\left|D^{2} g_{m}^{n}(u)\left(f_{k}, f_{l}\right)\right|^{2}=: c_{D^{2} G}^{2}<\infty
\end{aligned}
$$

where the $o_{u, h_{1}}^{n, m}, o_{u, h_{1}}$ have the same property as above.

Now we can verify the properties of the operator $G$ formulated in hypothesis $(\mathbf{G})$. It follows from (7.2) that

$$
\begin{aligned}
\sup _{u \in V_{-\delta}}\|G(u)\|_{L_{2}(V)}^{2} & =\sup _{u \in V_{-\delta}} \sum_{m=1}^{\infty}\left\|G(u) e_{m}\right\|^{2}=\sup _{u \in V_{-\delta}} \sum_{n, m=1}^{\infty}\left|\left(G(u) e_{m}\right)_{n}\right|^{2} \\
& =\sup _{u \in V_{-\delta}} \sum_{n, m=1}^{\infty}\left|g_{m}^{n}(u)\right|^{2}=c_{G}^{2} .
\end{aligned}
$$

Simple calculations show that (7.3), (7.4) imply that the operator $D G$ and $D^{2} G$ exist and are bounded. In fact, if $u, h \in V_{-\delta}$, then we have that

$$
\|G(u+h)-G(u)-D G(u) h\|_{L_{2}(V)}^{2}=\left(o\left(\|h\|_{V_{-\delta}}\right)\right)^{2}
$$

and

$$
\sup _{u \in V_{-\delta}}\|D G(u)\|_{L_{2}\left(V \times V_{-\delta}, V\right)}^{2}=\sup _{u \in V_{-\delta}} \sum_{m, k=1}^{\infty}\left\|D G(u)\left(e_{m}, f_{k}\right)\right\|^{2}=\sup _{u \in V_{-\delta}} \sum_{n, m, k=1}^{\infty}\left|D g_{m}^{n}(u) f_{k}\right|^{2}=c_{D G}^{2} .
$$

Now, using the boundedness of $D G$ we can prove the Lipschitz condition. Similarly, (7.4) implies that the operator $D^{2} G$ exists and is bounded. Using the boundedness of the second derivative of $G$ standard calculations give (4.1).

\section{Appendix}

We start this section by completing the proof of Lemma 5.4, although in the next result (item (i)) we prove a bit more.

Lemma 8.1. (i) Let $I_{4}, I_{5}$ be defined in (5.8). Then for any sufficient small $\varepsilon \geq 0$ such that $\beta^{\prime}-\hat{\beta}>\varepsilon$ we have

$$
\sup _{0 \leq p<q \leq t} \frac{\left\|A^{\varepsilon} I_{4}\right\|+\left\|A^{\varepsilon} I_{5}\right\|}{(q-p)^{\hat{\beta}}} \leq c t^{\beta^{\prime}-\hat{\beta}-\varepsilon}\left|\|\omega \mid\|_{\beta^{\prime}}\left(1+t^{\hat{\beta}} \mid\|u\| \|_{\hat{\beta},-\delta, 0, t}\right) .\right.
$$

(ii) Let $I_{2}, I_{3}$ be defined in (5.8) and let $0 \leq \varepsilon<\delta-1 / 2, \beta^{\prime}-\hat{\beta}>\varepsilon$. Then

$$
\sup _{0 \leq p<q \leq t} \frac{\left\|A^{\varepsilon} I_{2}\right\|+\left\|A^{\varepsilon} I_{3}\right\|}{(q-p)^{\hat{\beta}}} \leq c\left(t^{1-\hat{\beta}}+t^{\delta+1 / 2-\hat{\beta}-\varepsilon}\right)\|u\|_{C, 0, t}^{2} .
$$


Proof. Throughout the proof we will use frequently the properties (2.1), (2.2) and (5.4). We choose an $\alpha$ in the same conditions than in Lemma 5.3, that is, $1-\beta^{\prime}<\alpha<\hat{\beta}$. We also use the property $L_{2}(V) \subset L_{2}\left(V, V_{-\delta}\right)$. First, using the definition of the stochastic integral and the estimate (5.6),

$$
\begin{aligned}
& \sup _{0 \leq p<q \leq t} \frac{\left\|A^{\varepsilon} I_{4}\right\|}{(q-p)^{\hat{\beta}}} \leq \sup _{0 \leq p<q \leq t} \frac{1}{(q-p)^{\hat{\beta}}}\|\omega\|_{\beta^{\prime}} \int_{p}^{q}(q-r)^{\alpha+\beta^{\prime}-1}\left(\frac{\left\|S(q-r) A^{\varepsilon} A^{-\delta} G(u(r))\right\|_{L_{2}(V)}}{(r-p)^{\alpha}}\right. \\
& \left.\quad+\int_{p}^{r} \frac{\left\|(S(q-r)-S(q-\hat{r})) A^{\varepsilon} A^{-\delta} G(u(r))\right\|_{L_{2}(V)}}{(r-\hat{r})^{\alpha+1}} d \hat{r}+\int_{p}^{r} \frac{\left\|S(q-\hat{r}) A^{\varepsilon} A^{-\delta}(G(u(r))-G(u(\hat{r})))\right\|_{L_{2}(V)}}{(r-\hat{r})^{\alpha+1}} d \hat{r}\right) d r .
\end{aligned}
$$

The first term is estimated by

$$
\frac{\left\|S(q-r) A^{\varepsilon} A^{-\delta} G(u(r))\right\|_{L_{2}(V)}}{(r-p)^{\alpha}} \leq \frac{c c_{G}}{(r-p)^{\alpha}(q-r)^{\varepsilon}}
$$

and since $\alpha+\beta^{\prime}-\varepsilon>0$, we get

$$
\sup _{0 \leq p<q \leq t} \frac{c c_{G}}{(q-p)^{\hat{\beta}}}\left|\left\|\omega \left|\left\|\left.\right|_{\beta^{\prime}} \int_{p}^{q}(q-r)^{\alpha+\beta^{\prime}-\varepsilon-1}(r-p)^{-\alpha} d r \leq c \mid\right\| \omega\|\|_{\beta^{\prime}} t^{\beta^{\prime}-\hat{\beta}-\varepsilon} .\right.\right.\right.
$$

Concerning the second term, taking an appropriate $\alpha^{\prime}>\alpha$ such that $\alpha+\beta^{\prime}>\alpha^{\prime}+\varepsilon$, we have

$$
\int_{p}^{r} \frac{\left\|(S(q-r)-S(q-\hat{r})) A^{\varepsilon} A^{-\delta} G(u(r))\right\|_{L_{2}(V)}}{(r-\hat{r})^{\alpha+1}} d \hat{r} \leq \frac{c c_{G}}{(q-r)^{\alpha^{\prime}+\varepsilon}} \int_{p}^{r} \frac{(r-\hat{r})^{\alpha^{\prime}}}{(r-\hat{r})^{\alpha+1}} d \hat{r} \leq \frac{c c_{G}(r-p)^{\alpha^{\prime}-\alpha}}{(q-r)^{\alpha^{\prime}+\varepsilon}}
$$

and hence

$$
\sup _{0 \leq p<q \leq t} \frac{c c_{G}}{(q-p)^{\hat{\beta}}}\left\|\omega \left|\left\|_{\beta^{\prime}} \int_{p}^{q} \frac{(r-p)^{\alpha^{\prime}-\alpha}}{(q-r)^{\alpha^{\prime}+\varepsilon}}(q-r)^{\alpha+\beta^{\prime}-1} d r \leq c \mid\right\| \omega\|\|_{\beta^{\prime}} t^{\beta^{\prime}-\hat{\beta}-\varepsilon} .\right.\right.
$$

Finally, since $\hat{\beta}>\alpha$

$$
\begin{gathered}
\int_{p}^{r} \frac{\left\|S(q-\hat{r}) A^{\varepsilon} A^{-\delta}(G(u(r))-G(u(\hat{r})))\right\|_{L_{2}(V)}}{(r-\hat{r})^{\alpha+1}} d \hat{r} \leq c \int_{p}^{r} \frac{\left\|A^{-\delta}(G(u(r))-G(u(\hat{r})))\right\|_{L_{2}(V)}}{(r-\hat{r})^{\alpha+1}(q-\hat{r})^{\varepsilon}} d \hat{r} \\
\leq c c_{D G}\left|\left\|u \left|\left\|_{\hat{\beta},-\delta, 0, t} \frac{1}{(q-r)^{\varepsilon}} \int_{p}^{r} \frac{(r-\hat{r})^{\hat{\beta}}}{(r-\hat{r})^{\alpha+1}} d \hat{r} \leq c c_{D G}\right\|\|u \mid\| \|_{\hat{\beta},-\delta, 0, t} \frac{(r-p)^{\hat{\beta}-\alpha}}{(q-r)^{\varepsilon}},\right.\right.\right.
\end{gathered}
$$

and since $\beta^{\prime}+\alpha-\varepsilon>0$ we have

$$
\sup _{0 \leq p<q \leq t} \frac{c c_{D G}\left|\left\|u|\||_{\hat{\beta},-\delta, 0, t}\right.\right.}{(q-p)^{\hat{\beta}}}|||\omega|\left\|_{\beta^{\prime}} \int_{p}^{q}(q-r)^{\alpha+\beta^{\prime}-\varepsilon-1}(r-p)^{\hat{\beta}-\alpha} d r \leq c\left|\left\|\omega\left|\left\|\left.\right|_{\beta^{\prime}}\right\| u\|\|\right|_{\hat{\beta},-\delta, 0, t} t^{\beta^{\prime}-\varepsilon} .\right.\right.\right.
$$

Hence, we get that

$$
\sup _{0 \leq p<q \leq t} \frac{\left\|A^{\varepsilon} I_{4}\right\|}{(q-p)^{\hat{\beta}}} \leq c t^{\beta^{\prime}-\hat{\beta}-\varepsilon}\left|\|\omega \mid\| \|_{\beta^{\prime}}\left(1+t^{\hat{\beta}} \mid\|u\| \|_{\hat{\beta},-\delta, 0, t}\right) .\right.
$$

Thanks to the definition of the stochastic integral and the estimate (5.6) for $I_{5}$ we get

$$
\begin{aligned}
\sup _{0 \leq p<q \leq t} \frac{\left\|A^{\varepsilon} I_{5}\right\|}{(q-p)^{\hat{\beta}}} & \leq \sup _{0 \leq p<q \leq t} \frac{1}{(q-p)^{\hat{\beta}}}\|\omega\| \|_{\beta^{\prime}} \int_{0}^{p}(p-r)^{\alpha+\beta^{\prime}-1}\left(\frac{\left\|(S(q-r)-S(p-r)) A^{\varepsilon} A^{-\delta} G(u(r))\right\|_{L_{2}(V)}}{r^{\alpha}}\right. \\
& +\int_{0}^{r} \frac{\left\|(S(q-\hat{r})-S(p-\hat{r})) A^{\varepsilon} A^{-\delta}(G(u(r))-G(u(\hat{r})))\right\|_{L_{2}(V)}}{(r-\hat{r})^{\alpha+1}} d \hat{r} \\
& \left.+\int_{0}^{r} \frac{\left\|(S(q-r)-S(q-\hat{r})-S(p-r)+S(p-\hat{r})) A^{\varepsilon} A^{-\delta} G(u(r))\right\|_{L_{2}(V)}}{(r-\hat{r})^{\alpha+1}} d \hat{r}\right) d r \\
= & : \sup _{0 \leq p<q \leq t} \frac{1}{(q-p)^{\hat{\beta}}}\|\omega \mid\|_{\beta^{\prime}} \int_{0}^{p}(p-r)^{\alpha+\beta^{\prime}-1}\left(I_{5,1}+I_{5,2}+I_{5,3}\right) d r .
\end{aligned}
$$


We start with

$$
I_{5,1}=\frac{\left\|(S(q-p)-\mathrm{Id}) S(p-r) A^{\varepsilon} A^{-\delta} G(u(r))\right\|_{L_{2}(V)}}{r^{\alpha}} \leq \frac{c c_{G}(q-p)^{\hat{\beta}}}{(p-r)^{\hat{\beta}+\varepsilon} r^{\alpha}}
$$

and because $\alpha<1$ and $\alpha+\beta^{\prime}-\hat{\beta}-\varepsilon>0$, the term involving $I_{5,1}$ is estimated by

$$
\sup _{0 \leq p<q \leq t} \frac{c c_{G}}{(q-p)^{\hat{\beta}}}\left|\left\|\omega \left|\left\|\beta_{\beta^{\prime}}(q-p)^{\hat{\beta}} \int_{0}^{p}(p-r)^{\alpha+\beta^{\prime}-1-\hat{\beta}-\varepsilon} r^{-\alpha} d r \leq c \mid\right\| \omega\|\|_{\beta^{\prime}} t^{\beta^{\prime}-\hat{\beta}-\varepsilon} .\right.\right.\right.
$$

On the other hand,

$$
\begin{aligned}
I_{5,2} & =\int_{0}^{r} \frac{\left\|(S(q-p)-\mathrm{Id}) S(p-\hat{r}) A^{\varepsilon} A^{-\delta}(G(u(r))-G(u(\hat{r})))\right\|_{L_{2}(V)}}{(r-\hat{r})^{\alpha+1}} d \hat{r} \\
& \leq c c_{D G} \int_{0}^{r} \frac{(p-\hat{r})^{-\hat{\beta}-\varepsilon}(q-p)^{\hat{\beta}}\|u(r)-u(\hat{r})\|_{V_{-\delta}}}{(r-\hat{r})^{\alpha+1}} d \hat{r} \\
& \leq c c_{D G}\|\| u\|\|_{\hat{\beta},-\delta, 0, t}(p-r)^{-\hat{\beta}-\varepsilon}(q-p)^{\hat{\beta}} \int_{0}^{r} \frac{1}{(r-\hat{r})^{\alpha+1-\hat{\beta}}} d \hat{r} \\
& \leq c c_{D G}\|\| u \|_{\hat{\beta},-\delta, 0, t}(p-r)^{-\hat{\beta}-\varepsilon}(q-p)^{\hat{\beta}} r^{\hat{\beta}-\alpha},
\end{aligned}
$$

and thus

$$
\begin{aligned}
\sup _{0 \leq p<q \leq t} & \frac{1}{(q-p)^{\hat{\beta}}}\left|\|\omega \mid\|_{\beta^{\prime}} \int_{0}^{p}(p-r)^{\alpha+\beta^{\prime}-1} I_{5,2} d r\right. \\
& \leq c c_{D G}|||\omega|\|\|_{\beta^{\prime}}|||u| \|\left.\right|_{\hat{\beta},-\delta, 0, t} \sup _{\substack{0 \leq p<q \leq t \\
\beta^{\prime}}} \int_{0}^{p}(p-r)^{\alpha+\beta^{\prime}-1-\hat{\beta}-\varepsilon} r^{\hat{\beta}-\alpha} d r \\
& \leq c c_{D G}|||\omega|\|\|_{\beta^{\prime}}|||u| \|\left.\right|_{\hat{\beta},-\delta, 0, t^{\beta^{\prime}-\varepsilon}}
\end{aligned}
$$

Finally, taking $\alpha^{\prime}$ close enough to $\alpha$ such that $\alpha^{\prime}>\alpha$ and $\alpha+\beta^{\prime}>\alpha^{\prime}+\hat{\beta}+\varepsilon$ (for a small enough $\varepsilon$ ), applying the second part of $(2.3)$

$$
\begin{aligned}
I_{5,3} & \leq c \int_{0}^{r} \frac{(q-p)^{\hat{\beta}}(r-\hat{r})^{\alpha^{\prime}}(p-r)^{-\alpha^{\prime}-\hat{\beta}-\varepsilon}\left\|A^{-\delta} G(u(r))\right\|_{L_{2}(V)}}{(r-\hat{r})^{\alpha+1}} d \hat{r} \\
& \leq c c_{G}(q-p)^{\hat{\beta}}(p-r)^{-\alpha^{\prime}-\hat{\beta}-\varepsilon} \int_{0}^{r}(r-\hat{r})^{\alpha^{\prime}-\alpha-1} d \hat{r} \\
& \leq c c_{G}(q-p)^{\hat{\beta}}(p-r)^{-\alpha^{\prime}-\hat{\beta}-\varepsilon} r^{\alpha^{\prime}-\alpha},
\end{aligned}
$$

and hence

$$
\begin{aligned}
\sup _{0 \leq p<q \leq t} & \frac{1}{(q-p)^{\hat{\beta}}}\|\omega\| \|_{\beta^{\prime}} \int_{0}^{p}(p-r)^{\alpha+\beta^{\prime}-1} I_{5,3} d r \\
& \leq c c_{G}\|\| \omega\|\|_{\beta^{\prime}} \sup _{\substack{0 \leq p<q \leq t \\
0}}^{p}(p-r)^{\alpha+\beta^{\prime}-1-\alpha^{\prime}-\hat{\beta}-\varepsilon} r^{\alpha^{\prime}-\alpha} d r \\
& \leq c c_{G}\||| \omega \mid\|_{\beta^{\prime}} t^{\beta^{\prime}-\hat{\beta}-\varepsilon} .
\end{aligned}
$$

Taking into account the previous estimates we finally get

$$
\sup _{0 \leq p<q \leq t} \frac{\left\|A^{\varepsilon} I_{5}\right\|}{(q-p)^{\hat{\beta}}} \leq c t^{\beta^{\prime}-\hat{\beta}-\varepsilon}\left|\left\|\omega|\||_{\beta^{\prime}}\left(1+t^{\hat{\beta}} \mid\|u\| \|_{\hat{\beta},-\delta, 0, t}\right) .\right.\right.
$$

(ii) The proof of this part follows similarly to the estimates (5.10) and (5.11). In particular, for the estimate of $\left\|A^{\varepsilon} I_{2}\right\|$ we need to use the continuous embedding $V \subset V_{-\delta+\varepsilon+1 / 2}$, which holds true for small enough $\varepsilon \geq 0$ since $\delta \in(\hat{\beta}, 1)$. 
Corollary 8.2. (i) We derive (5.12) simply taking $\varepsilon=0$ in Lemma 8.1 (i).

(ii) As a consequence of Lemma 8.1 we can prove the inequality (6.7):

$$
\left.||\left|u_{n}\right|\right|_{\hat{\beta},-\hat{\delta}, 0, t} \leq c t^{\hat{\delta}-\hat{\beta}}\left\|u_{n}(0)\right\|+c\left(t^{1-\hat{\beta}}+t^{\hat{\delta}-1 / 2-\hat{\beta}}\right)\left\|u_{n}\right\|_{C, 0, t}^{2}+c\left|\left\|\omega_{n}|\||_{\beta^{\prime}} t^{\beta^{\prime}-\hat{\beta}-\varepsilon}\left(1+t^{\hat{\beta}}\left\|\left|u_{n}\right|\right\|_{\hat{\beta},-\hat{\delta}, 0, t}\right) .\right.\right.
$$

Note that, since $\hat{\delta}=\delta-\varepsilon$, now we have the splitting

$$
\begin{aligned}
& A^{-\hat{\delta}}\left(u_{n}(q)-u_{n}(p)\right)=A^{-\hat{\delta}}(S(q)-S(p)) u_{n}(0)+A^{\varepsilon} A^{-\delta+1 / 2} \int_{p}^{q} S(q-r) A^{-1 / 2} B\left(u_{n}(r), u_{n}(r)\right) d r \\
& +A^{\varepsilon} A^{-\delta+1 / 2} \int_{0}^{p}(S(q-r)-S(p-r)) A^{-1 / 2} B\left(u_{n}(r), u_{n}(r)\right) d r \\
& +A^{\varepsilon} A^{-\delta} \int_{p}^{q} S(q-r) G\left(u_{n}(r)\right) \omega_{n}^{\prime}(r) d r+A^{\varepsilon} A^{-\delta} \int_{0}^{p}(S(q-r)-S(p-r)) G\left(u_{n}(r)\right) \omega_{n}^{\prime}(r) d r \\
& =A^{\varepsilon} I_{1}+A^{\epsilon} I_{2}+A^{\varepsilon} I_{3}+A^{\epsilon} I_{4}+A^{\epsilon} I_{5},
\end{aligned}
$$

where for $j=2,3,4,5$ the $I_{j}$ agree with the integrals previously defined in (5.8). Firstly, similar to (5.9), we obtain that

$$
\sup _{0 \leq p<q \leq t} \frac{\left\|A^{\varepsilon} I_{1}\right\|}{(q-p)^{\hat{\beta}}} \leq \bar{c} t^{\hat{\delta}-\hat{\beta}}\left\|u_{n}(0)\right\|
$$

and by Lemma 8.1 (ii)

$$
\sup _{0 \leq p<q \leq t} \frac{\left\|A^{\varepsilon} I_{2}\right\|+\left\|A^{\varepsilon} I_{3}\right\|}{(q-p)^{\hat{\beta}}} \leq c\left(t^{1-\hat{\beta}}+t^{\hat{\delta}+1 / 2-\hat{\beta}}\right)\left\|u_{n}\right\|_{C, 0, t}^{2} .
$$

Secondly, on account of the fact that $L_{2}(V) \subset L_{2}\left(V, V_{-\delta}\right)$ and that $V_{-\hat{\delta}} \subset V_{-\delta}$, from assumption $(\mathbf{G})$ for instance we derive that

$$
\left\|A^{-\delta}\left(G\left(u_{1}\right)-G\left(u_{2}\right)\right)\right\|_{L_{2}(V)} \leq\left\|G\left(u_{1}\right)-G\left(u_{2}\right)\right\|_{L_{2}(V)} \leq c_{D G}\left\|u_{1}-u_{2}\right\|_{V_{-\delta}} \leq c\left\|u_{1}-u_{2}\right\|_{V_{-\hat{\delta}}},
$$

and therefore, following the same steps than in the proof of Lemma 8.1 (i) we can deduce

$$
\sup _{0 \leq p<q \leq t} \frac{\left\|A^{\varepsilon} I_{4}\right\|+\left\|A^{\varepsilon} I_{5}\right\|}{(q-p)^{\hat{\beta}}} \leq c t^{\beta^{\prime}-\hat{\beta}-\varepsilon}||\left|\omega_{n}\right|||_{\beta^{\prime}}\left(1+\left.t^{\hat{\beta}}\left\|\left|u_{n}\right|\right\|\right|_{\hat{\beta},-\hat{\delta}, 0, t}\right),
$$

and this concludes the proof of (6.7).

The rest of the Appendix section is devoted to the proof of Lemma 5.5, which relies upon several results that are proven below.

Lemma 8.3. Let $1 / 2<\hat{\beta}<\tilde{\beta}<\delta$ and suppose that $u \in C^{\tilde{\beta}}\left([0, T] ; V_{-\delta}\right)$. Then the mapping

$$
[s, T] \ni t \mapsto\||| u \mid\|_{\hat{\beta},-\delta, s, t}
$$

is continuous and

$$
\lim _{t \rightarrow s^{+}}\left|\left\|u|\||_{\hat{\beta},-\delta, s, t}=0 .\right.\right.
$$

Proof. We only consider here the case $s=0$. Let us define the following transformation of $u$ given by

$$
\hat{u}_{\hat{t}}(r)=\left\{\begin{array}{lll}
u(r) & : & r \leq \hat{t} \\
u(\hat{t}) & : & r \geq \hat{t}
\end{array}\right.
$$

Then for $0 \leq \hat{t}<t \leq T$

$$
\left\|\left.|| u||\right|_{\hat{\beta},-\delta, 0, t}-|||u|||_{\hat{\beta},-\delta, 0, \hat{t}}=\left.|||u|\right|_{\hat{\beta},-\delta, 0, t}-\right\||| \hat{u}_{\hat{t}}||_{\hat{\beta},-\delta, 0, t} \leq|\| u|||_{\hat{\beta},-\delta, \hat{t}, t} \leq c(t-\hat{t})^{\tilde{\beta}-\hat{\beta}}|||u|||_{\tilde{\beta},-\delta, 0, T}
$$

from which the desired continuity follows immediately. The convergence to 0 follows in the same way. 
Lemma 8.4. For positive continuous functions $a(t), b(t)$ consider

$$
Y=b(t)+a(t) Y^{2}
$$

and assume $4 a(t) b(t)<1$ for every $t \in\left[0, t_{1}\right]$, where $t_{1}>0$ is some positive number. Then there exist two real solutions $Y_{1}(t)<Y_{2}(t) \in \mathbb{R}^{+}$given by

$$
Y_{1}(t)=\frac{1}{2 a(t)}(1-\sqrt{1-4 a(t) b(t)}), \quad Y_{2}(t)=\frac{1}{2 a(t)}(1+\sqrt{1-4 a(t) b(t)})
$$

where $Y_{1}(t) \leq 2 b(t)$. Suppose in addition that $y(t) \geq 0$ is continuous on $\left[0, t_{1}\right]$ such that

$$
y(t) \leq b(t)+a(t) y(t)^{2}, \quad \lim _{t \rightarrow 0^{+}} y(t)=0,
$$

and that $\lim _{t \rightarrow 0^{+}} a(t)=0$. Then we have $y(t) \leq Y_{1}(t)$ on $\left[0, t_{1}\right]$.

Proof. It follows by Sohr [28] Page 317 that under the conditions of the lemma there exist real solutions $Y_{1}$, $Y_{2}$ satisfying the above conditions.

On the other hand, $y$ satisfies the above inequality if and only if $y(t) \leq Y_{1}(t)$ or $y(t) \geq Y_{2}(t)$. If $y(t) \geq Y_{2}(t)$ for some $t \in\left(0, t_{1}\right]$ then by the continuity of $y, Y_{2}$ and by the fact that $Y_{2}(t)>Y_{1}(t)$ on $\left(0, t_{1}\right]$, it follows that $y(t) \geq Y_{2}(t)$ on $\left[0, t_{1}\right]$. However, under the assumptions we have $\lim _{t \rightarrow 0^{+}} Y_{2}(t)=+\infty$ and this is a contradiction with respect to the behavior of $y$.

To simplify the presentation of the following technical result we assume that $T=1$. Moreover, in what follows, see Lemma 8.5 below, we shall consider inequalities of the type

$$
y(t) \leq d(t, x) y(t)+f(t, x)+h(t) y(t)^{2}, \quad t \in\left[0, t_{1}\right]
$$

where the increasing functions $d(\cdot, x), f(\cdot, x), h(\cdot)$ are defined by

$$
\begin{aligned}
& d(t, x)=c t^{\beta^{\prime}}+4 c^{3} t^{1+2 \beta^{\prime}}+2 c^{2} x t^{1+\beta^{\prime}}, \\
& f(t, x)=\bar{c} x t^{\delta-\hat{\beta}}+c x^{2} t^{1-\hat{\beta}}+c^{2} x t^{1+\beta^{\prime}-\hat{\beta}}+c^{3} t^{1+2 \beta^{\prime}-\hat{\beta}}+c t^{\beta^{\prime}-\hat{\beta}}, \\
& h(t)=4 c^{3} t^{1+2 \beta^{\prime}+\hat{\beta}} .
\end{aligned}
$$

Note that $d(t, x)$ and $f(t, x)$ depend on a positive parameter $x$. Furthermore, the constants $\bar{c}, c, c^{2}, c^{3}$ are coming from the estimates of Lemma 5.3 and Lemma 5.4, as we will show in Lemma 8.5 below. In particular, these constants are constricted such that they are including the value $\left.\||\omega|\|\right|_{\beta^{\prime}}=\left|\|\omega \mid\| \|_{\beta^{\prime}, 0,1}\right.$. In the following proof we need these constants with norms only for subintervals of $[0,1]$. However, using ||$|\omega|||_{\beta^{\prime}, 0,1}$ these constants can be chosen independently of the subinterval. In that result, depending on the value of $x$ we shall choose $t_{1}>0$ such that $d\left(t_{1}, x\right) \leq 1 / 2$. Then, defining $a(t):=2 h(t)$ and $b(t, x):=2 f(t, x)$ we can rewrite (8.1) as

$$
y(t) \leq b(t, x)+a(t) y(t)^{2}, \quad t \in\left[0, t_{1}\right]
$$

which looks like the inequality of Lemma 8.4. Let us emphasize that with the above choice $\lim _{t \rightarrow 0^{+}} a(t)=$ $\lim _{t \rightarrow 0^{+}} 2 h(t)=0$. In the next result we will also choose suitable values of $x$ such that the rest of assumptions of Lemma 8.4 also holds.

Lemma 8.5. Let $\left(\omega_{n}\right)_{n \in \mathbb{N}}$ be a sequence of piecewise linear continuous paths converging to $\omega$ in the sense of Remark 3.2, and let $\left(u_{n}\right)_{n \in \mathbb{N}}$ be a sequence of solutions for $(2.5)$ on $[0,1]$ driven by $\left(\omega_{n}\right)_{n \in \mathbb{N}}$ with initial condition $u_{0} \in V$. Then, there exist constants $K \geq \hat{K}>1$ (uniform in $n$ ) defining finitely many intervals $\left(I_{i}\right)_{i=1, \cdots, i^{*}}$ by

$$
I_{1}=\left[0, \frac{1}{\hat{K}}\right]=\left[\check{t}_{1}, \hat{t}_{1}\right], \cdots, I_{i}=\left[\hat{t}_{i-1}, \hat{t}_{i-1}+\frac{1}{K i}\right]=\left[\check{t}_{i}, \hat{t}_{i}\right]
$$

in such a way that on $I_{i}$ we have uniformly for any $n \in \mathbb{N}$

$$
\begin{gathered}
\left.\left\||| u_{n}\right\|\right|_{\hat{\beta},-\delta, I_{1}} \leq(\hat{K})^{\hat{\beta}},\left.\quad\left\|u_{n}\right\|\right|_{\hat{\beta},-\delta, I_{i}} \leq(K i)^{\hat{\beta}} \\
\left\|u_{n}\right\|_{C, I_{1}} \leq \frac{4 c \hat{K}^{1-\beta^{\prime}}}{1-\beta^{\prime}}, \quad\left\|u_{n}\right\|_{C, I_{i}} \leq \frac{4 c(K i)^{1-\beta^{\prime}}}{1-\beta^{\prime}}
\end{gathered}
$$


for $i=2, \cdots, i^{*}$. This constant $c$ in particular depends on $\|\mid \omega\|_{\beta^{\prime}, 0,1}$.

We point out that in the previous result $i^{*}$ is given by the condition $\check{t}_{i^{*}}<1=T \leq \hat{t}_{i^{*}}$, and in this case we set $\hat{t}_{i^{*}}=1$.

Proof. Let us denote by $x_{0}:=\max \left\{1,\left\|u_{0}\right\|\right\}, x_{1}(t):=\max \left\{1,\left\|u_{n}\right\|_{C, 0, t}\right\}$ and $y_{1}(t):=\left\|u_{n}|\||_{\hat{\beta},-\delta, 0, t}\right.$, for $t \in I_{1}=\left[0, \hat{t}_{1}\right]$, where $\hat{t}_{1}$ will be determined later. The inequality (5.5) implies

$$
\begin{aligned}
\left\|u_{n}(t)\right\|^{2} & \leq\left\|u_{0}\right\|^{2}+c t^{\beta^{\prime}}\left\|u_{n}\right\|_{C, 0, t}+c t^{\hat{\beta}+\beta^{\prime}}\left(1+\left\|u_{n}\right\|_{C, 0, t}\right)\left\|u_{n}\right\| \|_{\hat{\beta},-\delta, 0, t} \\
& \leq x_{0}^{2}+c t^{\beta^{\prime}}\left\|u_{n}\right\|_{C, 0, t}+c t^{\hat{\beta}+\beta^{\prime}}\left(1+\left\|u_{n}\right\|_{C, 0, t}\right)\|\| u_{n}\|\|_{\hat{\beta},-\delta, 0, t} .
\end{aligned}
$$

Moreover, since $x_{0} \geq 1$ we also have

$$
1 \leq x_{0}^{2}+c t^{\beta^{\prime}}\left\|u_{n}\right\|_{C, 0, t}+c t^{\hat{\beta}+\beta^{\prime}}\left(1+\left\|u_{n}\right\|_{C, 0, t}\right)\|\| u_{n}\|\|_{\hat{\beta},-\delta, 0, t} .
$$

Then

$$
\begin{aligned}
\max \left\{\left\|u_{n}\right\|_{C, 0, t}^{2}, 1\right\} & \leq x_{0}^{2}+c t^{\beta^{\prime}}\left\|u_{n}\right\|_{C, 0, t}+c t^{\hat{\beta}+\beta^{\prime}}\left(1+\left\|u_{n}\right\|_{C, 0, t}\right)\|\| u_{n}\|\|_{\hat{\beta},-\delta, 0, t} \\
& \leq x_{0}^{2}+c t^{\beta^{\prime}} \max \left\{1,\left\|u_{n}\right\|_{C, 0, t}\right\}+c t^{\hat{\beta}+\beta^{\prime}}\left(1+\max \left\{1,\left\|u_{n}\right\|_{C, 0, t}\right\}\right)\left\|u_{n}\right\| \|_{\hat{\beta},-\delta, 0, t}
\end{aligned}
$$

and therefore

$$
x_{1}^{2}(t) \leq x_{0}^{2}+c x_{1}(t) t^{\beta^{\prime}}+2 c x_{1}(t) y_{1}(t) t^{\hat{\beta}+\beta^{\prime}} .
$$

Note that in (8.3) we have used that $x_{1}(t) \geq 1$ and therefore the corresponding last term on the left hand side of (5.5) can be estimated as

$$
c t^{\hat{\beta}+\beta^{\prime}}\left(1+x_{1}(t)\right) y_{1}(t) \leq 2 c x_{1}(t) y_{1}(t) t^{\hat{\beta}+\beta^{\prime}} .
$$

Furthermore, since $t \in[0,1]$ and $\delta+1 / 2>1$, we have that $t^{\delta+1 / 2-\hat{\beta}}<t^{1-\hat{\beta}}$, and thus (5.7) implies

$$
y_{1}(t) \leq \bar{c} x_{0} t^{\delta-\hat{\beta}}+c x_{1}^{2}(t) t^{1-\hat{\beta}}+c t^{\beta^{\prime}-\hat{\beta}}+c y_{1}(t) t^{\beta^{\prime}} .
$$

Now combining (8.3) with (8.4) we get

$$
y_{1}(t) \leq \bar{c} x_{0} t^{\delta-\hat{\beta}}+c\left(x_{0}^{2}+c x_{1}(t) t^{\beta^{\prime}}+2 c x_{1}(t) y_{1}(t) t^{\hat{\beta}+\beta^{\prime}}\right) t^{1-\hat{\beta}}+c t^{\beta^{\prime}-\hat{\beta}}+c y_{1}(t) t^{\beta^{\prime}} .
$$

In addition, from (8.3) the following estimate holds

$$
x_{1}(t) \leq \frac{c t^{\beta^{\prime}}+2 c y_{1}(t) t^{\hat{\beta}+\beta^{\prime}}}{2}+\sqrt{\frac{\left(c t^{\beta^{\prime}}+2 c y_{1}(t) t^{\hat{\beta}+\beta^{\prime}}\right)^{2}+4 x_{0}^{2}}{4}} \leq c t^{\beta^{\prime}}+2 c y_{1}(t) t^{\hat{\beta}+\beta^{\prime}}+x_{0}
$$

and plugging this into (8.5) we finally arrive at

$$
y_{1}(t) \leq d\left(t, x_{0}\right) y_{1}(t)+f\left(t, x_{0}\right)+h(t) y_{1}(t)^{2}, \quad t \in I_{1}=\left[0, \hat{t}_{1}\right],
$$

where the functions have been defined in (8.2). Then, taking $a(t)=2 h(t)$ and $b\left(t, x_{0}\right)=2 f\left(t, x_{0}\right)$ there exists a $K_{1} \geq 1$ such that for any $\hat{K} \geq K_{1}$ and $\hat{t}_{1}=\hat{K}^{-1}$ we have

$$
d\left(\hat{K}^{-1}, x_{0}\right) \leq \frac{1}{2}, \quad b\left(\hat{K}^{-1}, x_{0}\right) \leq \frac{(\hat{K})^{\hat{\beta}}}{2}, \quad 4 a\left(\hat{K}^{-1}\right) b\left(\hat{K}^{-1}, x_{0}\right)<1 .
$$

Hence, we have the conditions Lemma 8.4 and as a consequence we claim that $y_{1}\left(\hat{t}_{1}\right) \leq(\hat{K})^{\hat{\beta}}=\hat{t}_{1}^{-\hat{\beta}}$. Let us fix such a $\hat{K}$ such that in addition $x_{0} \leq c \hat{K}^{1-\beta^{\prime}} /\left(1-\beta^{\prime}\right)$. Then, from (8.6), simply using the general notation for constants $c$, we get

$$
x_{1}\left(\hat{t}_{1}\right) \leq 3 c \hat{t}_{1}^{\beta^{\prime}}+x_{0} \leq \frac{4 c \hat{K}^{1-\beta^{\prime}}}{1-\beta^{\prime}}=: \hat{x}_{1} .
$$

Now we can repeat the same arguments than above in each interval $I_{i}, i=2,3, \cdots$, by doing the corresponding suitable changes. In order to do that we need to rewrite the estimates (5.5) and (5.7) in those intervals. In particular, in $I_{i}$ we have to take as initial condition $u_{n}\left(\hat{t}_{i-1}\right)$ and $t$ can be estimated by the length of 
the interval $I_{i}$ which is nothing but $(K i)^{-1}$. Taking $x_{i}(t):=\max \left\{1,\left\|u_{n}\right\|_{C, \hat{t}_{i-1}, t}\right\}$ with $x_{i}\left(\hat{t}_{i-1}\right) \geq 1$ and $y_{i}(t):=\left.\left|\| u_{n}\right|\right|_{\hat{\beta},-\delta, \hat{t}_{i-1}, t}$, for $t \in I_{i}$, for induction we assume

$$
x_{i-1}\left(t_{i-1}\right) \leq 3 c \sum_{j=1}^{i-1}(K j)^{-\beta^{\prime}}+x_{0} \leq \frac{4 c(K(i-1))^{1-\beta^{\prime}}}{1-\beta^{\prime}}=: \hat{x}_{i-1}
$$

and choose $K>\hat{K}$ such that for $i=2,3, \cdots$

$$
\begin{aligned}
d\left((K i)^{-1}, \hat{x}_{i-1}\right) & =c(K i)^{-\beta^{\prime}}+4 c^{3}(K i)^{-1-2 \beta^{\prime}}+2 c^{2} \hat{x}_{i-1}(K i)^{-\beta^{\prime}-1} \\
& \leq c K^{-\beta^{\prime}}+4 c^{3} K^{-1-2 \beta^{\prime}}+\frac{8 c^{3}}{1-\beta^{\prime}} K^{1-\beta^{\prime}} K^{-\beta^{\prime}-1} \leq o\left(K^{-\varepsilon}\right) \leq \frac{1}{2} \\
f\left((K i)^{-1}, \hat{x}_{i-1}\right) & =\bar{c} \hat{x}_{i-1}(K i)^{\hat{\beta}-\delta}+c \hat{x}_{i-1}^{2}(K i)^{\hat{\beta}-1}+c^{2} \hat{x}_{i-1}(K i)^{-1+\hat{\beta}-\beta^{\prime}}+c^{3}(K i)^{-1-2 \beta^{\prime}+\hat{\beta}}+c(K i)^{-\beta^{\prime}+\hat{\beta}} \\
& \leq C o\left(K^{-\varepsilon}\right)(K i)^{\hat{\beta}} \leq \frac{(K i)^{\hat{\beta}}}{4}
\end{aligned}
$$

for a constant $C$ and an sufficiently small $\varepsilon>0$ independent of $K$ and $i$. For example, for the critical term in the expression of $f$ given for the quadratic term, we have that

$$
c \hat{x}_{i-1}^{2}(K i)^{\hat{\beta}-1} \leq \frac{16 c^{3}}{\left(1-\beta^{\prime}\right)^{2}} K^{2-2 \beta^{\prime}-1+\hat{\beta}} i^{2-2 \beta^{\prime}-1+\hat{\beta}} \leq \frac{16 c^{3}}{\left(1-\beta^{\prime}\right)^{2}} K^{1-2 \beta^{\prime}}(K i)^{\hat{\beta}} \leq \operatorname{Co}\left(K^{-\varepsilon}\right)(K i)^{\hat{\beta}}
$$

where this last inequality is true since $\beta^{\prime} \in(1 / 2,1)$.

Again, for $a(t)=2 h(t), b\left(t, \hat{x}_{i-1}\right)=2 f\left(t, \hat{x}_{i-1}\right)$, choosing $K$ sufficiently large such that

$$
4 a\left((K i)^{-1}\right) b\left((K i)^{-1}, \hat{x}_{i-1}\right) \leq 64 c^{3}(K i)^{-1-2 \beta^{\prime}-\hat{\beta}} \frac{(K i)^{\hat{\beta}}}{4}<1
$$

we obtain by Lemma 8.3 and Lemma 8.4 that $y_{i}\left(\hat{t}_{i}\right) \leq(K i)^{\hat{\beta}}$. If we denote $\hat{t}_{i}-\check{t}_{i}=: \Delta t_{i}=(K i)^{-1}$ the previous inequality can be rewriten as $y_{i}\left(\hat{t}_{i}\right) \leq\left(\Delta t_{i}\right)^{-\hat{\beta}}$, and similar to (8.6)

$$
\begin{aligned}
x_{i}\left(\hat{t}_{i}\right) & \leq c \Delta t_{i}^{\beta^{\prime}}+2 c y_{i}\left(\hat{t}_{i}\right) \Delta t_{i}^{\hat{\beta}+\beta^{\prime}}+x_{i-1}\left(\hat{t}_{i-1}\right) \leq 3 c \Delta t_{i}^{\beta^{\prime}}+x_{i-1}\left(\hat{t}_{i-1}\right) \\
& \leq x_{0}+3 c \sum_{j=1}^{i}(K j)^{-\beta^{\prime}} \leq x_{0}+3 c K^{-\beta^{\prime}} \int_{0}^{i} r^{-\beta^{\prime}} d r \leq x_{0}+\frac{3 c K^{-\beta^{\prime}} i^{1-\beta^{\prime}}}{1-\beta^{\prime}} \leq \frac{4 c(K i)^{1-\beta^{\prime}}}{1-\beta^{\prime}}=: \hat{x}_{i}
\end{aligned}
$$

and therefore we obtain that $x_{i}\left(\hat{t}_{i}\right) \leq \hat{x}_{i}$.

Finally we present the proof of Lemma 5.5:

Proof. Following the steps of Proposition 5.4 we prove that each $u_{n} \in C^{\tilde{\beta}}\left([0, T] ; V_{-\delta}\right)$ with $1 / 2<\hat{\beta}<$ $\tilde{\beta}$. Then we apply Lemmas $8.3-8.5$ obtaining that this sequence is uniformly bounded in $C^{\hat{\beta}}\left([0, T] ; V_{-\delta}\right) \cap$ $C([0, T] ; V)$.

\section{ACKNOWLEDGEMENTS}

Hakima Bessaih's research was supported in part by NSF grant DMS-1418838.

\section{REFERENCES}

[1] D. Barbato, M. Barsanti, H. Bessaih, F. Flandoli, Some rigorous results on a stochastic GOY model, Jour. Stat. Phys., 125(3): 677-716, 2006.

[2] H. Bessaih, B. Ferrario, Invariant Gibbs measures of the energy for shell models of turbulence; the inviscid and viscous cases, Nonlinearity, 25: 1075-1097, 2012.

[3] H. Bessaih, B. Ferrario, Invariant measures of Gaussian type for 2D turbulence, Jour. Stat. Phys., 149(2): 259-283, 2012.

[4] H. Bessaih, M.J. Garrido-Atienza, B. Schmalfuss, Random attractors for stochastic shell equations driven by fractional Brownian motion, in preparation.

[5] T. Cass, Z. Qian, J. Tudor, Non-linear evolution equations driven by rough paths. Stochastic analysis and applications to finance, 1-18, Interdiscip. Math. Sci., 13, World Sci. Publ., Hackensack, NJ, 2012.

[6] Y. Chen, H. Gao, M. J. Garrido-Atienza, B. Schmalfuss, Pathwise solutions of SPDEs and random dynamical systems, Discrete and continuous dynamical systems, series A, 34(1): 79-98, 2014.

[7] P. Constantin, B. Levant, E.S. Titi, Analytic study of shell models of turbulence, Phys. D., 219(2): 120-141, 2006. 
[8] P. Constantin, B. Levant, E.S. Titi, Regularity of inviscid shell models of turbulence, Phys. Rev. E., (3) 75 (1), 016304,10 pp, 2007.

[9] G. Da Prato, J. Zabczyk, Stochastic equations in infinite dimensions, Cambridge University Press, Cambridge, 1992.

[10] M.A. Diop, M.J.Garrido-Atienza, Retarded stochastic evolution systems driven by fractional Brownian motion with Hurstparameter $H>1 / 2$, Nonlinear Analysis: TMA., 97:15-29, 2014.

[11] L.H. Duc, S. Siegmund and B. Schmalfuss, A note of the generation of random dynamical systems for fractional stochastic delay differential equations, Stochastic and Dynamics, 15(3), 1550018, 2015.

[12] L. Fang, P. Sundar, F. G. Viens, Two-dimensional stochastic Navier-Stokes equations with fractional Brownian noise, Random Oper. Stoch. Equ., 21(2): 135-158, 2013.

[13] P. Friz and M. Hairer, A Course on Rough Paths, with an introduction to regularity structures, Universitext. Springer, Cham, 2014.

[14] P. Friz and N. Victoir. Multidimensional Stochastic Processes as Rough Paths. Theory and Applications, Cambridge Studies of Advanced Mathematics Vol. 120. Cambridge University Press, 2010.

[15] H. Gao, M. J. Garrido-Atienza, B. Schmalfuss, Random attractors for stochastic evolution equations driven by fractional Brownian motion, SIAM J. Math. Anal. 46(4): 2281-2309, 2014.

[16] M.J. Garrido-Atienza, K. Lu and B. Schmalfuss, Random dynamical systems for stochastic partial differential equations driven by a fractional Brownian motion, Discrete and continuous dynamical systems, series B, 14 (2): 473-493, 2010.

[17] M. J. Garrido-Atienza, B. Maslowski and B. Schmalfuss, Random attractors for stochastic equations driven by a fractional Brownian motion, International Journal of Bifurcation and Chaos, 20(9): 1-22, 2010.

[18] E. B. Gledzer, System of hydrodynamic type admitting two quadratic integrals of motion. Dokl. Akad. Nauk SSSR 209: 1046-1048, 1973 (Engl. Transl.: Sov. Phys.Dokl. 18: 216-217, 1973).

[19] H. Kunita, Stochastic Flows and Stochastic Differential Equations. Cambridge University Press, 1990.

[20] V. S. L'vov, E. Podivilov, A. Pomyalov, I. Procaccia, D. Vandembroucq, Improved shell model of turbulence, Physical Review E, 58, 1811-1822, 1998.

[21] B. Maslowski and D. Nualart, Evolution equations driven by a fractional Brownian motion, J. Funct. Anal., 202(1):277-305, 2003.

[22] D. Nualart and A. Răşcanu, Differential equations driven by fractional Brownian motion, Collect. Math., 53(1):55-81, 2002.

[23] D. Nualart and P.A. Vuillermot, Variational solutions for partial differential equations driven by a fractional noise, Journal of Functional Analysis, 232:390-454, 2006.

[24] K. Ohkitani, M. Yamada, Lyapunov Spectrum of a Chaotic Model of Three-Dimensional Turbulence, J. Phys. Soc. Jpn., 56, 4210-4213, 1987.

[25] A. Pazy, Semigroups of Linear Operators and Applications to Partial Differential Equations. Springer Applied Mathematical Series. Springer-Verlag, Berlin, 1983.

[26] S.G. Samko, A.A. Kilbas, O.I. Marichev, Fractional integrals and derivatives: Theory and applications, Gordon and Breach Science Publishers (Switzerland and Philadelphia, Pa., USA), 1993.

[27] G.R. Sell, Y. You, Dynamics of Evolutionary Equations, Springer Applied Mathematical Series. Springer-Verlag, Berlin, 2002.

[28] H. Sohr, The Navier-Stokes equations. An elementary functional analytic approach, Birkhäuser Advances Texts, Birkhäuser Varlag, Basel-Boston-Berlin, 2001.

[29] R. Temam, Navier-Stokes Equations. Theory and numerical analysis, Studies in Mathematics and its Applications 2, (NorthHolland Publishing Co), 1979.

[30] M. I. Vishik and A. V. Fursikov, Mathematical Problems of Statistical Hydromechanics, Kluwer Academic Publishers, 1988.

[31] L.C. Young, An integration of Hölder type, connected with Stieltjes integration, Acta Math., 67, 251-282, 1936.

[32] M. Zähle, Integration with respect to fractal functions and stochastic calculus. I, Probab. Theory Related Fields, 111(3):333$374,1998$.

(Hakima Bessaih) Department of Mathematics, University of Wyoming, Laramie 82071 USA

E-mail address, Hakima Bessaih: bessaih@uwyo.edu

(María J. Garrido-Atienza) Dpto. Ecuaciones Diferenciales y Análisis Numérico, Universidad de Sevilla, Apdo. de Correos 1160, 41080-Sevilla, Spain

E-mail address, María J. Garrido-Atienza: mgarrido@us.es

(Björn Schmalfuss) Institut für Stochastik, Friedrich Schiller Universität Jena, Ernst Abbe Platz 2, 77043, Jena, Germany

E-mail address, Björn Schmalfuss: bjoern.schmalfuss@uni-jena.de 